TRANSACTIONS OF THE

AMERICAN MATHEMATICAL SOCIETY

Volume 362, Number 12, December 2010, Pages 6231-6259

S 0002-9947(2010)04943-8

Article electronically published on August 3, 2010

\title{
THE EIGENVALUES OF THE LAPLACIAN ON DOMAINS WITH SMALL SLITS
}

\author{
LUC HILLAIRET AND CHRIS JUDGE
}

\begin{abstract}
We introduce a small slit into a planar domain and study the resulting effect upon the eigenvalues of the Laplacian. In particular, we show that as the length of the slit tends to zero, each real-analytic eigenvalue branch tends to an eigenvalue of the original domain. By combining this with our earlier work (2009), we obtain the following application: The generic multiply connected polygon has a simple spectrum.
\end{abstract}

\section{INTRODUCTION}

In this paper we study the following singular perturbation problem. Let $\Omega \subset \mathbb{R}^{2}$ be a bounded open set having Lipschitz boundary. Remove from $\Omega$ the horizontal slit, $\Sigma_{t}=\left\{(x, y)\left|y=y_{0},\right| x-x_{0} \mid \leq t\right\}$, centered at the point $\left(x_{0}, y_{0}\right) \in \Omega$. Standard analytic perturbation theory shows that the eigenvalues of the Laplacian on the slit domain $\Omega \backslash \Sigma_{t}$ depend analytically on $t$ for $t>0$. (See Theorem 4.2.) The main result of our study is the following.

Theorem 1.1. Let $t \mapsto E_{t}$ be a real-analytic eigenvalue branch of the Laplacian acting on $L^{2}\left(\Omega \backslash \Sigma_{t}\right)$ with either Dirichlet or Neumann boundary conditions. Then $E_{t}$ converges to an eigenvalue of the Laplacian acting on $L^{2}(\Omega)$ as $t$ tends to 0 .

We remark that the convergence of real-analytic eigenbranches is much subtler than the convergence of ordered eigenvalues. For example, as $t$ tends to zero, the $k^{\text {th }}$ eigenvalue of the Laplacian on the rectangle $[0, t] \times[0,1 / t]$ tends to zero, but infinitely many analytic eigenvalue branches limit to infinity. Note that our proof that each eigenbranch in Theorem 1.1 has a finite limit is more involved than the proof that ordered eigenvalues limit to an eigenvalue of $\Omega$. (Compare $\$ 3$ and $₫ 6$ )

This work began as part of a study of the Laplace spectrum of degenerating translation surfaces. Indeed, the collapse of a slit is one of the typical degenerations of a translation surface [EMZ03]. Theorem 1.1] deals with the simplest case of such a degeneration. The slit degeneration of a translation surface is analogous to the well-studied 'neck pinching' degeneration of hyperbolic surfaces, and our overall strategy for proving Theorem 1.1 mirrors the strategy employed in Wlp92 and Jdg02.

The strategy is to control the negative variation of the logarithmic derivative, $\partial_{t} \log \left(E_{t}\right)$, for small $t$. A well-known formula (Proposition 4.6) relates $\partial_{t} E_{t}$ to certain quadratic forms defined as integrals over the domain. Not unexpectedly, one need only consider the contribution to these integrals that comes from a small

Received by the editors March 3, 2008 .

2010 Mathematics Subject Classification. Primary 58C40; Secondary 58J37, 35P20.

(C)2010 American Mathematical Society Reverts to public domain 28 years from publication 
neighborhood of the slit (Corollary 4.11). A judicious choice of coordinates in a neighborhood of the slit allows one to reduce the analysis of the quadratic forms to a family of 1 -dimensional problems indexed by $i=0,1,2,3 \ldots$ A simple convexity estimate (Lemma 5.3) provides for control of the 1-dimensional contributions for $i>(2 t)^{2} \cdot E_{t}$, where $2 t$ is the width of the slit. We first show that $t^{2} \cdot E_{t}$ is bounded and then use convexity for $i$ large and compactness for $i$ small to find that $t^{2 k} \cdot E_{t}$ tends to zero for some $k<1$. Thus convexity applies to all $i>0$, and a special estimate can be made for the case $i=0$.

Elliptical coordinates in a neighborhood of the slit are particularly well-suited for our purpose. Define $(r, \theta)$ implicitly by

$$
\begin{aligned}
x-x_{0} & =\sqrt{r^{2}+t^{2}} \cdot \cos (\theta), \\
y-y_{0} & =r \cdot \sin (\theta) .
\end{aligned}
$$

The slit then corresponds exactly to the locus $r=0$, and the level sets $r=$ const correspond to ellipses that surround the slit. If, in turn, one sets $r=t \sinh (z)$, then the equation $\Delta \psi=E \cdot \psi$ is separable in the variables $(z, \theta)$. The solutions to the resulting ordinary differential equations are called Mathieu functions. Appendix $\mathrm{A}$ contains the basic facts about these functions that we use here. We note that the work of Y. Colin de Verdiere CdV87. called our attention to the usefulness of these coordinates when considering slits, a fact of which mathematical physicists have long been aware MrsRbn38, MrsFsh.

We use Theorem 1.1 to extend the following generic simplicity result to polygons that are not necessarily simply connected.

Theorem 1.2 ([HlrJdg09]). If $n \geq 4$, then for almost every simply connected $n$-gon, the Dirichlet (resp. Neumann) Euclidean Laplacian on P has simple eigenvalues.

For example, we consider multiply connected polygons with $n$ exterior vertices 1 In $\S 7$, we prove the following:

Theorem 1.3. If $n \geq 4$, then almost every multiply connected polygon has a simple Laplace spectrum.

In Theorem 1.3 we assume that the boundary conditions are either Neumann on every edge or Dirichlet on every edge. We also consider 'mixed' boundary conditions in the sense that the condition on each boundary segment is either Dirichlet or Neumann.

Theorem 1.4. For each combinatorial choice of mixed Dirichlet-Neumann boundary conditions, almost every simply connected polygon with $n \geq 4$ vertices has a simple Laplace spectrum.

This latter theorem may be extended to multiply connected polygons with some mixed boundary conditions. It should be noted, however, that our method doesn't give generic simplicity for every type of mixed boundary conditions in the case of multiply connected polygons. For instance, we cannot handle a slit with Dirichlet conditions on one side and Neumann conditions on the other.

We now outline the contents of this paper. In $\S 2$ we precisely describe the eigenvalue problem on a slit domain and give a fuller description of elliptical coordinates.

\footnotetext{
${ }^{1}$ One can regard such a polygon as the result of removing finitely many simply connected polygons from a simply connected $n$-gon.
} 
In $\S 3$ we prove that sequences of eigenfunctions with uniformly bounded eigenvalues have convergent subsequences as the slit parameter $t$ tends to zero. In $\S 4$ we apply Kato's theory of analytic perturbations to show that the eigenvalues and eigenfunctions depend analytically on $t$. We also derive variational formulas for these eigenvalues and prove that uniform estimates on the logarithmic derivative can be 'localized' to a neighborhood of the slit. In $\S 5$ we prove a convexity result for 'radial' Mathieu functions. In $\S 6$, we prove Theorem 1.1 according to the strategy described above. In $\S 7$, we precisely state and then prove results concerning the spectral simplicity of polygons including Theorems 1.3 and 1.4

\section{The eigenvalue Problem On A SLIT DOMAIN}

Let $\Omega \subset \mathbb{R}^{2}$ be a compact domain with Lipschitz boundary, and let $\Sigma$ be a disjoint union of line segments contained in the interior of $\Omega$. The complement $\Omega \backslash \Sigma$ is not a domain with Lipschitz boundary, and hence one must take care in defining the eigenvalue problem. The purpose of this section is to describe a resolution that will be useful to us. We refer the reader to Gri] for a complementary discussion of the analysis of singular domains.

To define the eigenvalue problem, we will regard a slit domain as the compact manifold with Lipschitz boundary obtained by completing $\Omega \backslash \Sigma$ with respect to the length metric. To be more precise, we define the distance $d(x, y)$ between $x$ and $y$ to be the infimum of lengths of rectifiable paths $\gamma:[0,1] \rightarrow \Omega \backslash \Sigma$ that join $x$ to $y$. Note that $d(x, y)$ equals the Euclidean distance between $x$ and $y$ iff $x$ and $y$ belong to a convex subset of $\Omega \backslash \Sigma$.

Definition 2.1. The slit domain $\Omega_{\Sigma}$ is the metric completion of $\Omega \backslash \Sigma$ with respect to $d$. We will refer to $\Omega_{\Sigma} \backslash(\Omega \backslash \Sigma)$ as the slit.

Although $\Omega_{\Sigma}$ is not isometric to a subdomain of $\mathbb{R}^{2}$, it is naturally a compact Riemannian manifold with Lipschitz boundary. To see this, one can use elliptical coordinates to define a chart in a neighborhood of the slit.

2.1. Elliptical coordinates in a neighborhood of the slit. Let $S^{1}=\mathbb{R} /(2 \pi \mathbb{Z})$, and for each $t \geq 0$, define $\phi_{t}: \mathbb{R}^{+} \times S^{1} \rightarrow \mathbb{R}^{2}$ by

$$
\phi_{t}(r, \theta)=\left(\sqrt{r^{2}+t^{2}} \cdot \cos (\theta), r \sin (\theta)\right) .
$$

This injective map sends each circle $\{r\} \times S^{1}$ onto an ellipse with foci at $( \pm t, 0)$. We have $\phi_{t}\left(\mathbb{R}^{+} \times S^{1}\right)=\mathbb{R}^{2} \backslash \Sigma_{t}$, where $\Sigma_{t}=[-t, t] \times\{0\}$. By regarding $\mathbb{R}^{2} \backslash \Sigma_{t}$ as a subset of $\mathbb{R}_{\Sigma_{t}}^{2}$, the map $\phi_{t}$ extends to a smooth diffeomorphism from $[0, \infty) \times S^{1}$ onto $\mathbb{R}_{\Sigma_{t}}^{2}$. Abusing notation slightly, we will use $\phi_{t}$ to denote this extension.

In these elliptical coordinates $(r, \theta)$ about the slit $\Sigma_{t}$, the gradient operator takes the form

$$
\nabla_{t} w=\frac{r^{2}+t^{2}}{r^{2}+t^{2} \sin ^{2}(\theta)} \cdot \frac{\partial w}{\partial r} \cdot \partial_{r}+\frac{1}{r^{2}+t^{2} \sin ^{2}(\theta)} \cdot \frac{\partial w}{\partial \theta} \cdot \partial_{\theta},
$$

and Lebesgue measure is expressed as

$$
d m_{t}=\frac{r^{2}+t^{2} \sin ^{2}(\theta)}{\left(r^{2}+t^{2}\right)^{\frac{1}{2}}} d r d \theta
$$


The eigenvalue problem is unchanged if we rotate and/or translate the domain $\Omega$ and the slit $\Sigma \subset \Omega$ simultaneously. Thus, in the sequel, we will often make the following assumption.

Assumption 2.2. We assume that $\Sigma=[-t, t] \times\{0\}$.

Now choose $r_{0}>0$ so that the ball $B\left(\overrightarrow{0}, 2 r_{0}\right)$ is contained in $\Omega$. Then for $t<r_{0}$, the restriction of $\phi_{t}^{-1}$ to $\left[0,2 r_{0}\right) \times S^{1}$ provides a chart in a neighborhood of the slit.

2.2. The eigenvalue problem. Let $d m$ denote Lebesgue measure. For each smooth function on the manifold $\Omega_{\Sigma}$ define

$$
N(u)=\int_{\Omega}|u|^{2} d m
$$

and

$$
q(u)=\int_{\Omega}|\nabla u|^{2} d m
$$

Given a measurable subset, $D$, of the boundary of $\Omega_{\Sigma}$, define $H_{D}^{1}\left(\Omega_{\Sigma}\right)$ to be the completion of

$$
\left\{u \in C^{\infty}\left(\Omega_{\Sigma}\right) \mid u(m)=0, \forall m \in D\right\}
$$

with respect to the norm

$$
u \mapsto q^{\frac{1}{2}}(u)+N^{\frac{1}{2}}(u) .
$$

The form $q$ extends to a closed quadratic form on $H_{D}^{1}\left(\Omega_{\Sigma}\right)$, and the form $N$ extends to a closed quadratic form on $L^{2}\left(\Omega_{\Sigma}, d m\right)$. In the sequel we will let $\mathfrak{n}(\cdot, \cdot)$ (resp. $\mathfrak{q}(\cdot, \cdot))$ denote the polarization of $N$ (resp. q).

A function $\psi \in H_{D}^{1}\left(\Omega_{\Sigma}\right)$ is an eigenfunction with eigenvalue $E$ if and only if

$$
\mathfrak{q}(\psi, v)=E \cdot \mathfrak{n}(\psi, v)
$$

for all $v \in H_{D}^{1}\left(\Omega_{\Sigma}\right)$. Integration by parts and standard elliptic estimates give that $\psi$ is smooth in $\Omega_{\Sigma}$ with $\Delta \psi=E \cdot \psi$, where $\Delta$ is the Laplacian acting on smooth functions on $\mathbb{R}^{2}$. Moreover, we have

(1) $\psi(m)=0$ for all $m \in \operatorname{Int}(D)$ (Dirichlet conditions), and

(2) $\nu(\psi)(m)=0$ for all $m \in \operatorname{Int}\left(\partial \Omega_{\Sigma} \backslash D\right)$ (Neumann conditions).

Here $\nu$ denotes the outward normal derivative along the boundary, $\partial \Omega_{\Sigma}$, of $\Omega_{\Sigma}$. Note that in the sequel $D$ will essentially be a union of segments.

\section{Convergence of Eigenfunctions with Bounded eigenvalues}

Let $\Omega$ be a domain that contains the origin $\{0\}$, and let $t_{n}>0$ be a sequence with $\lim _{n \rightarrow \infty} t_{n}=0$. For sufficiently large $n$, the segment $\Sigma_{t_{n}}=\left[-t_{n}, t_{n}\right] \times\{0\}$ lies in the interior of $\Omega$. For each $n \in \mathbb{N}$, let $D_{n}$ be a measurable subset of the boundary of the slit domain $\Omega_{\Sigma_{t_{n}}}$ such that $D=D_{n} \cap \partial \Omega$ does not depend on $n$. Let $\psi_{n}$ be a normalized eigenfunction of $q$ on $H_{D_{n}}^{1}\left(\Omega_{\Sigma_{t_{n}}}\right)$ with eigenvalue $E_{n}$. In this section we prove the following:

Theorem 3.1. If $E_{n}$ is a bounded sequence, then a subsequence $\psi_{n(k)}$ converges in $L^{2}(\Omega)$ to an eigenfunction $\psi$ of $q$ on $H_{D}^{1}(\Omega)$ with eigenvalue $E=\lim _{k \rightarrow \infty} E_{n(k)}$. Moreover, for every neighborhood $U$ of the slit and every $j \in \mathbb{N}$, the sequence $\psi_{n(k)}$ converges to $\psi$ in $C^{j}(\Omega \backslash U)$. 
Proof. Let $U$ be a neighborhood of the slit. Each $\psi_{n}$ is an eigenfunction of $\Delta$ with eigenvalue $E_{n}$, and hence for each $n$ such that $\Sigma_{t_{n}} \subset U$ we have

$$
\int_{\Omega \backslash U}\left|\psi_{n} \cdot \Delta^{j} \psi_{n}\right| d m=E_{n}^{j} \int_{\Omega \backslash U}\left|\psi_{n}\right|^{2} d m .
$$

Thus, using Gårding's inequality and the fact that $\psi_{n}$ is normalized, we find that

$$
\left\|\psi_{n}\right\|_{j} \leq C \cdot\left(E_{n}^{k}+1\right),
$$

where $\|\cdot\|_{j}$ is the norm associated to the Sobolev space $H^{j}(\Omega \backslash U)$. Since by assumption $E_{n}$ is a bounded sequence, we have that for each $j$, the sequence $n \mapsto$ $\left\|\psi_{n}\right\|_{j}$ is bounded.

Using a diagonalization argument, we find a function $\psi$ and a subsequence of $\psi_{n}$ (still denoted $\psi_{n}$ ) such that for every $j$ and every neighborhood $U$ of the slit, $\psi_{n}$ converges to $\psi$ in the $H^{j}(\Omega \backslash U)$ norm. Thus, by the Sobolev embedding theorem, $\psi_{n}$ converges to $\psi$ in $C^{k}(\Omega \backslash U)$ for every $k \in \mathbb{N}$.

By assumption, the $\psi_{n}$ have $L^{2}$-norm equal to 1 . Thus, given $\epsilon>0$, Lemma 3.2 below applies to give $N_{1}>0$ and $r^{*}$ so that if $n>N_{1}$, then

$$
\int_{B}\left|\psi_{n}\right|^{2} d m \leq \frac{\epsilon}{6}
$$

where $B$ is some fixed ball centered at 0 and included in the neighborhood $U_{t_{n}, r *}$ for $n>N_{1}$. Hence, by Fatou's Lemma, we also have

$$
\int_{B}|\psi|^{2} d m \leq \frac{\epsilon}{6}
$$

The functions $\psi_{n}$ converge uniformly to $\psi$ on the complement of $B$, and hence there exists $N_{2}$ so that if $n>N_{2}$, then

$$
\int_{\Omega \backslash U}\left|\psi-\psi_{n}\right|^{2} d m<\frac{\epsilon}{3} .
$$

It follows that $\psi_{n}$ converges to $\psi$ in $L^{2}(\Omega)$. In particular, $\psi$ is $L^{2}$-normalized and hence is nontrivial.

Finally, we show that $\psi$ is an eigenfunction. To do this we adapt an argument from CdV82. Define the distribution $T$ by $T(\phi)=\int_{\Omega} \psi \cdot \phi d m$. Then $\Delta^{\prime} T \in$ $H^{-2}(\Omega)$, where $\Delta^{\prime}$ denotes the distributional Laplacian. If the support of $\phi$ does not contain the origin, then $\Delta^{\prime} T(\phi)=E \cdot T(\phi)$, and hence the singular support of $\Delta^{\prime} T-E T$ is contained in $\{0\}$. Hence there exists $S \in H^{-2}(\Omega)$ with $\operatorname{supp}(S) \subset\{0\}$ such that

$$
\Delta^{\prime} T=E \cdot T+S .
$$

It follows that there exists $C$ such that $S=C \cdot \delta$, where $\delta(\phi)=\phi(0)$.

Let $G$ be the distribution defined by $G(\phi)=\int_{\Omega} L \cdot \phi d m$, where $L(x, y)=$ $\ln \left(\sqrt{x^{2}+y^{2}}\right) \chi(x, y)$ and where $\chi$ is some smooth cut-off function near 0 (say for instance in the ball $B$ ). A direct computation shows that $\Delta^{\prime} G-\delta$ is in $L^{2}(\Omega)$, and hence it follows from above that $\Delta^{\prime}(T-C \cdot G)$ is also in $L^{2}(\Omega)$ and hence, by elliptic regularity, $T-C \cdot G \in H^{2}(\Omega)$.

It suffices to show that $C=0$. On the one hand, by Fatou's Lemma, we have

$$
\int_{\Omega}|\nabla \psi|^{2} d m \leq \liminf _{n \rightarrow \infty} \int_{\Omega}\left|\nabla \psi_{n}\right|^{2} d m=E,
$$


and on the other hand,

$$
\int_{\Omega}|\nabla L|^{2} d m \geq 2 \pi \int_{0}^{\epsilon}\left(\partial_{r} \ln (r)\right)^{2} r d r=\infty,
$$

where $B(0, \epsilon) \subset \Omega$ is a ball centered at the origin. Therefore, $T-C \cdot G \notin H^{1}(\Omega)$ unless $C=0$.

Lemma 3.2. Given $\epsilon>0$ and $E_{0}>0$, there exists $r^{*}>0$ such that if $u$ is an eigenfunction of $q$ on $H_{D}^{1}\left(\Omega_{\Sigma_{t}}\right)$ with eigenvalue $E \leq E_{0}$, then for all $t<r^{*}$ we have

$$
\int_{U_{t, r^{*}}}|u|^{2} d m \leq \epsilon \int_{\Omega}|u|^{2} d m,
$$

where $U_{t, r^{*}}$ is the elliptical neighborhood of the slit $\Sigma_{t}$ of radius $r^{*}$.

Proof. For positive $r$ and $\rho$, we have

$$
\begin{aligned}
|u(r+\rho, \theta)-u(r, \theta)| & \leq \int_{0}^{\rho}\left|\partial_{r} u(r+s, \theta)\right| d s \\
& \leq \sqrt{\rho} \cdot\left(\int_{0}^{\rho}\left|\partial_{r} u(r+s, \theta)\right|^{2} d s\right)^{\frac{1}{2}}
\end{aligned}
$$

by the Cauchy-Schwarz inequality. From this we find that

$$
\frac{1}{2} \cdot|u(r, \theta)|^{2} \leq|u(r+\rho, \theta)|^{2}+\rho \int_{0}^{\rho}\left|\partial_{r} u(r+s, \theta)\right|^{2} d s .
$$

Given $r_{1}>0$, define $U_{s}=\left\{r \mid s \leq r \leq r_{1}+s\right\} \times S^{1}$. Using (11) and (2), we find that if $s \geq 0$, then

$$
\begin{aligned}
\int_{U_{0}}\left|\partial_{r} u(r+s, \theta)\right|^{2} d m & \leq \int_{U_{0}}\left|\partial_{r} u(r+s, \theta)\right|^{2}\left(r^{2}+t^{2}\right)^{\frac{1}{2}} d \theta d r \\
& \leq \int_{U_{0}}\left|\partial_{r} u(r+s, \theta)\right|^{2}\left((r+s)^{2}+t^{2}\right)^{\frac{1}{2}} d \theta d r \\
& =\int_{U_{s}}\left|\partial_{r} u(r, \theta)\right|^{2}\left(r^{2}+t^{2}\right)^{\frac{1}{2}} d \theta d r . \\
& \leq \int_{U_{s}}|\nabla u(r, \theta)|^{2} d m .
\end{aligned}
$$

Thus, since $u$ is an eigenfunction with eigenvalue $E$,

$$
\rho \int_{0}^{\rho} \int_{U_{0}}\left|\partial_{r} u(r+s, \theta)\right|^{2} d m d s \leq \rho^{2} E \int_{\Omega}|u|^{2} d m .
$$

If we define $\rho_{1}=\sqrt{E_{0} / 2 \epsilon}$, then it follows from (3) and (4) that

$$
\frac{1}{2} \int_{U_{0}}|u(r, \theta)|^{2} d m \leq \int_{U_{0}}\left|u\left(r+\rho_{1}, \theta\right)\right|^{2} d m+\frac{\epsilon}{4} \int_{\Omega}|u|^{2} d m,
$$

where $U_{0}$ is the ellipse of radius $r_{1}$.

Let

$$
r^{*}=\min \left\{\rho_{1}, \frac{\rho_{1}}{\sqrt{2}} \cdot \frac{\epsilon}{4}\right\} .
$$

If $t \leq r^{*}$ and $r \leq r^{*}$, then

$$
\rho_{1} \cdot\left(r^{2}+t^{2}\right)^{\frac{1}{2}} \leq \sqrt{2} \cdot \rho_{1} \cdot r^{*} \leq \sqrt{2} \cdot r^{*} \cdot\left(r+\rho_{1}\right),
$$


and hence it follows that

$$
\left(r^{2}+t^{2}\right)^{\frac{1}{2}} \leq \frac{\epsilon}{4} \cdot\left(\left(r+\rho_{1}\right)^{2}+t^{2}\right)^{\frac{1}{2}} .
$$

From this we find that for $t, r \leq r^{*}$,

$$
\frac{r^{2}+t^{2} \sin ^{2} \theta}{\left(r^{2}+t^{2}\right)^{\frac{1}{2}}} \leq \frac{\epsilon}{4} \cdot \frac{\left(r+\rho_{1}\right)^{2}+t^{2} \sin ^{2} \theta}{\left(\left(r+\rho_{1}\right)^{2}+t^{2}\right)^{\frac{1}{2}}} .
$$

Set $r_{1}=r^{*}$. Using (2) and (6), we find that

$$
\begin{aligned}
\int_{U_{0}}\left|u\left(r+\rho_{1}, \theta\right)\right|^{2} d m & \leq \frac{\epsilon}{4} \int_{U_{0}}\left|u\left(r+\rho_{1}, \theta\right)\right|^{2} \frac{\left(r+\rho_{1}\right)^{2}+t^{2} \sin ^{2} \theta}{\left(\left(r+\rho_{1}\right)^{2}+t^{2}\right)^{\frac{1}{2}}} d \theta d r \\
& =\frac{\epsilon}{4} \int_{U_{\rho_{1}}}|u(r, \theta)|^{2} \frac{r^{2}+t^{2} \sin ^{2} \theta}{\left(r^{2}+t^{2}\right)^{\frac{1}{2}}} d \theta d r \\
& \leq \frac{\epsilon}{4} \int_{\Omega}|u(r, \theta)|^{2} d m .
\end{aligned}
$$

By combining this estimate with (5), we obtain the lemma.

\section{Analyticity, Variational Formulae, AND LOCALIZATION}

In this section we use standard perturbation theory to show that the eigenvalues and eigenfunctions depend analytically on $t$ for $t>0$. We then derive basic formulae relating the derivative of an eigenbranch to the derivatives of the quadratic forms $q$ and $N$. We show that for small $t$ the dominant terms in the derivative fomulae can be localized to a neighborhood of the slit. Finally, we evaluate these local formulae on an elliptical neighborhood of the slit.

4.1. Pulling back to elliptical coordinates. Standard analytic perturbation theory Kato applies to a family of quadratic forms on a fixed Hilbert space. For this reason we will modify the family quadratic forms $q$ and $N$ but in such a way that we obtain an equivalent eigenvalue problem.

Of the various possible approaches, we choose to modify the map $\phi_{t}$ so that the inverse image of $\Omega_{\Sigma_{t}}$ is constant in $t$. The fixed Hilbert space will then be $L^{2}$ on this inverse image with respect to the pull-back of Lebesgue measure.

Let $r_{0}$ be as in $\oint_{2}$. Namely, $B\left(\overrightarrow{0}, 2 r_{0}\right) \subset \Omega$. Let $\sigma: \mathbb{R} \rightarrow \mathbb{R}$ be a smooth, positive, decreasing function such that $\sigma(x)=1$ if $|x| \leq 1$ and $\sigma(x)=0$ if $|x| \geq 2$. Define $\tilde{\phi}_{t}: \mathbb{R} \times S^{1} \rightarrow \mathbb{R}^{2}$ by

$$
\tilde{\phi}_{t}(r, \theta)=\left(\sqrt{r^{2}+t^{2} \cdot \sigma\left(r / r_{0}\right)} \cdot \cos (\theta), r \sin (\theta)\right) .
$$

By construction, $\tilde{\phi}_{t}$ defines a smooth diffeomorphism from $[0, \infty) \times \mathbb{R}$ onto $\mathbb{R}_{\Sigma_{t}}^{2}$.

If $t<r_{0}$, then the set $M=\tilde{\phi}_{t}^{-1}\left(\Omega_{t}\right)$ does not depend on $t$. We pull back the Dirichlet energy functional and the $L^{2}$-norm on $\Omega$ to functionals on $M$.

For computational convenience, we express the change of variables in the language of Riemannian geometry. For each $t$, define the Riemannian metric

$$
g_{t}=\tilde{\phi}_{t}^{*}\left(d x^{2}+d y^{2}\right)
$$

on $[0, \infty) \times S^{1}$. In particular, $\tilde{\phi}$ is a Riemannian isometry from $\left(\Omega_{t}, d x^{2}+d y^{2}\right)$ onto $\left(M, g_{t}\right)$.

Let $d m_{t}$ denote the Riemannian measure: $d m_{t}=\left(\tilde{\phi}_{t}\right)_{*}^{-1}(d x d y)$. The space, $L^{2}(M)$, of functions that are square integrable with respect to $d m_{t}$ does not depend 
on $t$. On the other hand, the associated norm does depend on $t$. Let $N_{t}$ denote the quadratic form 2

$$
N_{t}(u)=\int_{M}|u|^{2} d m_{t} .
$$

In the sequel we will let $\mathfrak{n}_{t}(\cdot, \cdot)$ denote the polarization of $N_{t}$.

Let $\nabla_{t}$ denote the Riemannian gradient for $\left(M, g_{t}\right)$. Namely, we have $g_{t}\left(\nabla_{t} u, X\right)$ $=d u(X)$ for every smooth function $u: M \rightarrow \mathbb{R}$ and vector field $X$ on $M$. For smooth functions $u: M \rightarrow \mathbb{R}$ define

$$
q_{t}(u)=\int_{M} g_{t}\left(\nabla_{t} u, \nabla_{t} u\right) d m_{t} .
$$

Given a measurable subset $D$ in the boundary of $M$, define $H_{D}^{1}(M)$ to be the completion with respect to the norm

$$
u \mapsto q_{t}(u)^{\frac{1}{2}}+N_{t}(u)^{\frac{1}{2}}
$$

on the set of smooth functions on $M$ that vanish on $D$. Since the equivalence class of this norm is independent of $t>0$, the completion does not depend on $t$. The form $q_{t}$ extends to a closed quadratic form on $H_{D}^{1}(M)$. In the sequel we will let $\mathfrak{q}_{t}(\cdot, \cdot)$ denote the polarization of $q_{t}$.

Proposition 4.1. $\psi$ is an eigenfunction of the quadratic form $q$ on $H_{\phi_{t}(D)}^{1}\left(\Omega_{\Sigma_{t}}\right)$ with respect to $N$ on $L^{2}\left(\Omega_{\Sigma_{t}}\right)$ with eigenvalue $E$ if and only if $u=\tilde{\phi}_{t}^{*}(\psi)$ is an eigenfunction of $q_{t}$ on $H_{D}^{1}(M)$ with respect to $N_{t}$ on $L^{2}(M)$ with eigenvalue $E$.

Proof. This follows from a straightforward accounting of the change of variables given by $\tilde{\phi}_{t}$.

\subsection{Analyticity of eigenvalues and eigenfunctions.}

Proposition 4.2. The eigenvalues and eigenfunctions of $q_{t}$ with respect to $N_{t}$ vary real-analytically. To be precise: $\forall k \in \mathbb{N}, \exists$ real-analytic paths $\psi_{k}:\left(0, t_{0}\right] \rightarrow H_{D}^{1}(M)$ and $E_{k}:\left(0, t_{0}\right] \rightarrow \mathbb{R}$ such that

(a) $\mathfrak{q}_{t}\left(\psi_{k}(t), v\right)=E_{k}(t) \cdot \mathfrak{n}_{t}\left(\psi_{k}(t), v\right)$ for all $v \in H_{D}^{1}(M)$,

(b) $N_{t}\left(\psi_{k}(t)\right)=1$, and

(c) the span of $\left\{\psi_{k}(t) \mid k \in \mathbb{N}\right\}$ is dense in $L^{2}(M)$.

Proof. Apply standard analytic perturbation theory. In particular, both $q_{t}$ and $N_{t}$ are holomorphic families of quadratic forms of type (a) as in §VII.4 Kato. The eigenvalue problem is of generalized form as in §VII.6 [Kato. (See especially Remark VII.6.2 Kato and the discussion on page 419.) The analogue of Remark VII.4.22 Kato gives the claim.

Remark 4.3. We will use the expression eigenbranch to designate the mapping $t \rightarrow\left(\psi_{k}(t), E_{k}(t)\right)$ and will use the expression normalized eigenbranch if, in addition, $N_{t}\left(\psi_{k}(t)\right)=1$. By extension, the term eigenbranch may also refer to either the eigenvalue or the eigenvector singly. Since we will be dealing with one eigenbranch at a time, the index $k$ will be systematically dropped.

\footnotetext{
${ }^{2}$ This quadratic form is defined on functions on $M$ and hence is not the same as the quadratic form $N$ defined in 2 On the other hand, these forms differ by pull-back by $\phi_{t}$, and the context will make clear which is being used.
} 
Corollary 4.4. The Neumann (resp. Dirichlet) eigenvalues of the Laplacian on $\Omega_{\Sigma_{t}}$ depend analytically on $t \in\left(0, t_{0}\right]$.

Proof. Combine Proposition 4.1, Proposition 4.2, and the discussion in 92

\subsection{Variational formulae.}

Notation 4.5. A dot above a quantity will indicate differentiation with respect to $t$. For example, $\dot{E}$ indicates the first derivative of an eigenvalue branch $E_{t}$. In what follows, we will often suppress the dependence of $q, \psi$, and $E$ on $t$ from the notation.

We begin with a well-known, general variational formula.

Proposition 4.6. We have

$$
\dot{E} \cdot N(\psi)=\dot{q}(\psi)-E \cdot \dot{N}(\psi) .
$$

Proof. Substitution of $\dot{\psi}$ for $v$ in part (a) of Proposition 4.2 gives

$$
\mathfrak{q}(\psi, \dot{\psi})=E \cdot \mathfrak{n}(\psi, \dot{\psi}) .
$$

By differentiating part (a) of Proposition 4.2, we obtain

$$
\dot{\mathfrak{q}}(\psi, v)+\mathfrak{q}(\dot{\psi}, v)=\dot{E} \cdot \mathfrak{n}(\psi, v)+E \cdot(\dot{\mathfrak{n}}(\psi, v)+\mathfrak{n}(\dot{\psi}, v))
$$

for all $v$. By substituting $\psi$ for $v$ and using (9), we find that

$$
\dot{q}(\psi, \psi)=\dot{E} \cdot N(\psi)+E \cdot \dot{N}(\psi) .
$$

In the case where $q$ is the Dirichlet energy associated to a family of Riemannian metrics, the quantities in Proposition 4.6 can be expressed in terms of the first variation of the metric and the associated Riemannian measure.

Proposition 4.7. Let $t \rightarrow g_{t}$ be a real-analytic family of Riemannian metrics on $M$, and suppose that $q$ is defined by (8). Then we have

$$
\dot{q}(u)=-\int_{M} \dot{g}(\nabla u, \nabla u) d m+\int_{M} g(\nabla u, \nabla u) d \dot{m}
$$

and

$$
\dot{N}(u)=\int_{M}|u|^{2} \dot{d m}
$$

Proof. By differentiating (8) we have

$$
\dot{q}(u)=\int_{M} \dot{g}(\nabla u, \nabla u) d m+2 \int_{M} g(\nabla u, \dot{\nabla} u) d m+\int_{M} g(\nabla u, \nabla u) \dot{d m} .
$$

By definition, $g(\nabla u, X)=X(f)$ for all vector fields $X$, and hence we have

$$
\dot{g}(\nabla u, X)+g(\dot{\nabla} u, X)=0 .
$$

In particular, if $X=\nabla u$, then we have

$$
\int_{M} \dot{g}(\nabla u, \nabla u) d m+\int_{M} g(\dot{\nabla} u, \nabla u) d m=0 .
$$

Substitution into the formula for $\dot{q}$ gives the first formula. The second formula follows from (7). 
The following lemma will be used to translate estimates on the logarithmic derivative of an eigenbranch into statements concerning the convergence of the eigenbranch.

Lemma 4.8. If there exists $t_{0}$ and a continuous positive function $\rho:\left(0, t_{0}\right] \rightarrow \mathbb{R}^{+}$ such that

$$
\partial_{t} E_{t} \geq-\rho(t) \cdot E_{t}
$$

for all $t \in\left(0, t_{0}\right]$, then the function

$$
F(t)=\exp \left(-\int_{t}^{t_{0}} \rho(s) d s\right) \cdot E_{t}
$$

converges as $t$ tends to zero. In particular, if $\rho$ is integrable, then $E_{t}$ converges as $t$ tends to zero.

Proof. We have

$$
F^{\prime}(t)=\left(\partial_{t} E_{t}+\rho(t) \cdot E\right) \cdot \exp \left(-\int_{t}^{t_{0}} \rho(s) d s\right) \geq 0,
$$

and hence $F$ is increasing. Since $F$ is nonnegative, the claim follows.

Using this lemma and Proposition 4.6 we have the following corollary.

Corollary 4.9. Suppose that there exists a constant $C$ such that for all $u \in H^{1}(M)$ we have

$$
\dot{q}(u) \geq-C \cdot q(u)
$$

and

$$
\dot{N}(u) \leq C \cdot N(u)
$$

Then $E_{t}$ converges as $t$ tends to 0.

Proof. From Proposition 4.6 we have $\dot{E} \geq-2 C \cdot E$. Thus we can apply Lemma 4.8 with $\rho \equiv 2 C$.

4.4. A localization principle. Unfortunately, inequalities (11) and (12) do not hold true for the singular perturbation that we consider here. We now show, however, that these inequalities do hold for all $u$ with support outside of a neighborhood of the slit (Proposition 4.10).

To state this result in a convenient form, we introduce the following notation. Let $U$ be a measurable set 3 For $w \in H^{1}$, we denote by $q_{U}(w)$ the 'restriction' of $q$ to $U$. That is,

$$
q_{U}(w)=\int_{U}|\nabla w|^{2} d m
$$

We will use analogous notation for the quadratic forms $\dot{q}, N$, and $\dot{N}$.

Proposition 4.10. Let $U \subset \Omega$ be a neighborhood of the slit. There exists a constant $C_{U}$ such that for any $w \in H^{1}$,

$$
\dot{q}_{M \backslash U}(w) \geq-C_{U} \cdot q(w)
$$

and

$$
\dot{N}_{M \backslash U}(w) \leq C_{U} \cdot N(w) .
$$

\footnotetext{
${ }^{3}$ In the sequel, $U$ will often be an elliptical neighborhood of the slit.
} 
Proof. Let $S M$ denote the unit tangent bundle to $M$ (with respect to $g_{t_{0}}$ ), and for each small $t$, define $F_{t}: S M \rightarrow \mathbb{R}$ by

$$
F_{t}(X)=\frac{\frac{d}{d t} g_{t}(X, X)}{g_{t}(X, X)} .
$$

The restriction of $g_{t}$ to $\Omega \backslash U$ is real-analytic for $t \in\left[0, t_{0}\right]$, and hence

$$
C_{1}=\sup \left\{F_{t}(X) \mid t \in\left[0, t_{0}\right] \text { and } X \in S(\Omega \backslash U)\right\}
$$

is finite. By homogeneity of $g$ and $\dot{g}$ in each tangent space, we have $\dot{g}(X, X) \leq$ $C_{1} \cdot g(X, X)$ for all $X \in T(\Omega \backslash U)$. Therefore,

$$
\int_{M \backslash U} \dot{g}(\nabla w, \nabla w) d m \leq C_{1} \int_{M \backslash U} g(\nabla w, \nabla w) d m .
$$

Let $G_{t}:\left[0, r_{0}\right] \times M \rightarrow \mathbb{R}$ be defined by

$$
G_{t}(p)=\frac{\frac{d}{d t} d m_{t}}{d m_{t}}(p),
$$

where $d m_{t}$ is the Riemannian measure associated to $g_{t}$. Since $d m_{t}$ restricted to $\Omega \backslash U$ depends real-analytically on $t \in\left[0, t_{0}\right]$,

$$
C_{2}=\sup \left\{|f(t, X)| \mid t \in\left[0, r_{0}\right] \text { and } X \in \Omega \backslash U\right\}
$$

is finite. It follows that

$$
\int_{M \backslash U} w^{2} \dot{d m} \leq C_{2} \int_{M \backslash U} w^{2} d m
$$

and

$$
\int_{M \backslash U} g(\nabla w, \nabla w) d \dot{m} \geq-C_{2} \int_{M \backslash U} g(\nabla w, \nabla w) d m .
$$

We use Proposition 4.7 and set $C_{U}=C_{1}+C_{2}$. The result then follows.

Corollary 4.11. Let $U$ be a neighborhood of the slit. Suppose that there exist functions $\alpha, \beta, \gamma:[0, \infty) \rightarrow[0, \infty)$ such that the following holds for an eigenbranch $\psi$ :

and

$$
\dot{q}_{U}(\psi) \geq-\alpha(t) \cdot q_{U}(\psi)-\beta(t) \cdot E \cdot N_{U}(\psi)
$$

Then there exists $C^{\prime}>0$ such that

$$
\dot{N}_{U}(\psi) \leq \gamma(t) \cdot N_{U}(\psi)
$$

$$
\dot{E} \geq-\left(C^{\prime}-\alpha(t)-\beta(t)-\gamma(t)\right) E .
$$

Proof. We have

$$
\begin{aligned}
\dot{q}(\psi) & =\dot{q}_{M \backslash U}(\psi)+\dot{q}_{U}(\psi) \\
& \geq-C \cdot q(\psi)-\alpha \cdot q_{U}(\psi)-\beta \cdot E \cdot N_{U}(\psi) \\
& \geq-(C+\alpha) \cdot q(\psi)-\beta \cdot E \cdot N(\psi) \\
& \geq-(C+\alpha+\beta) \cdot E \cdot N(\psi),
\end{aligned}
$$

where the constant $C$ comes from Proposition 4.10. Similarly, we find that

$$
\dot{N}_{U}(\psi) \leq(C+\gamma) \cdot N_{U}(\psi) .
$$

The estimate then follows from Proposition 4.6 with $C^{\prime}=2 C$. 
Remark 4.12. Proposition 4.10 and Corollary 4.11 represent a localization principle in the sense that an estimate of the logarithmic derivative of $E_{t}$ has been reduced to the study of the functionals $\dot{q}$ and $\dot{N}$ in a small neighborhood of the slit. This principle applies more generally to singular perturbation problems in which the 'singular support' of the perturbation is small.

4.5. Evaluation on the ellipse. We now let $U$ be the elliptical neighborhood of the slit of radius $r_{0}$ and evaluate $q_{U}$ and $N_{U}$. By using the expression for the gradient (11) and the Lebesgue measure (2) in elliptical coordinates, we find that

$$
q_{U}(v)=\int_{U}\left|\partial_{r} v\right|^{2}\left(r^{2}+t^{2}\right)^{\frac{1}{2}} d r d \theta+\int_{U}\left|\partial_{\theta} v\right|^{2} \frac{d r d \theta}{\left(r^{2}+t^{2}\right)^{\frac{1}{2}}}
$$

and

$$
N_{U}(v)=\int_{U}|v|^{2} \frac{\left(r^{2}+t^{2} \sin ^{2}(\theta)\right)}{\left(r^{2}+t^{2}\right)^{\frac{1}{2}}} d r d \theta .
$$

By Proposition 4.7 or direct computation, we find that

$$
\dot{q}_{U}(v)=t \int_{U}\left|\partial_{r} v\right|^{2} \frac{d r d \theta}{\left(r^{2}+t^{2}\right)^{\frac{1}{2}}}-t \int_{U}\left|\partial_{\theta} v\right|^{2} \frac{d r d \theta}{\left(r^{2}+t^{2}\right)^{\frac{3}{2}}}
$$

and

$$
\dot{N}_{U}(v)=t \int_{U}|v|^{2} \frac{\left(\left(2 \sin ^{2}(\theta)-1\right) r^{2}+t^{2} \sin ^{2}(\theta)\right)}{\left(r^{2}+t^{2}\right)^{\frac{3}{2}}} d r d \theta .
$$

It will prove convenient to make a change of variables.

Notation 4.13. In the following, we let

- $r=t \cdot \sinh x$,

- $Y_{t}=\sinh ^{-1}\left(r_{0} / t\right)$, and

- $U_{t}=\left[0, Y_{t}\right] \times S^{1}$.

With this change of coordinates, the formulae above become

$$
\begin{aligned}
& q_{U}(v)=\int_{U_{t}}\left|\partial_{x} v\right|^{2} d x d \theta+\int_{U_{t}}\left|\partial_{\theta} v\right|^{2} d x d \theta, \\
& N_{U}(v)=t^{2} \int_{U_{t}}|v|^{2}\left(\sinh ^{2} x+\sin ^{2}(\theta)\right) d x d \theta, \\
& \dot{q}_{U}(v)=\frac{1}{t} \int_{U_{t}} \frac{\left|\partial_{x} v\right|^{2}}{\cosh ^{2} x} d x d \theta-\frac{1}{t} \int_{U_{t}} \frac{\left|\partial_{\theta} v\right|^{2}}{\cosh ^{2} x} d x d \theta,
\end{aligned}
$$

and

$$
\dot{N}_{U}(v)=t \int_{U_{t}}|v|^{2}\left(\sin ^{2}(\theta)-\cos ^{2}(\theta) \tanh ^{2}(x)\right) d x d \theta .
$$

From (15) and (16) we find that

$$
\dot{q}_{U}(v) \geq-\frac{1}{t} \int_{U_{t}} \frac{\left|\partial_{\theta} v\right|^{2}}{\cosh ^{2} x} d x d \theta
$$

and

$$
\dot{N}_{U}(v) \leq t \int_{U_{t}}|v|^{2} \sin ^{2}(\theta) d x d \theta \leq t \int_{U_{t}}|v|^{2} d x d \theta .
$$




\section{Mathieu FUnCtions AND INTEGRAL EStimates}

In this section we provide estimates that will be used to control ratios such as $\dot{N} / N$ and $\dot{q} / q$ in the next section.

5.1. Separation of variables. E. Mathieu [Mth68] observed that one can apply the method of separation of variables to the eigenvalue problem, $\Delta \psi=E \cdot \psi$, on an ellipse in $\mathbb{R}^{2}$. He made a detailed study of the solutions to the resulting ordinary differential equations, solutions that now bear his name. To perform the separation of variables, we use the same change of variables as in the preceding section, i.e. we set

$$
r=t \cdot \sinh (x)
$$

Let $h \in \mathbb{R}$. The operator

$$
-\frac{\partial^{2}}{\partial \theta^{2}}+h^{2} \cos ^{2}(\theta)
$$

acting on $L^{2}(\mathbb{R} /(2 \pi \mathbb{Z}), d \theta)$ has the discrete spectrum

$$
0 \leq b_{0}(h) \leq b_{1}(h) \leq b_{2}(h) \leq \cdots .
$$

For $h \neq 0$, the spectrum is simple MrsFsh. Let $v_{i, h}$ denote the $L^{2}$-normalized eigenfunction associated to $b_{i}(h)$. We will call $v_{i, h}$ the $i^{\text {th }}$ angular Mathieu function. In the following we will often suppress the dependence of $v_{i, h}$ on $h$ from the notation.

For the convenience of the reader, we prove the following in Appendix $\mathrm{A}$

Proposition 5.1. Suppose that $\Delta \psi=E \cdot \psi$ on the ellipse of radius $r_{0}=t \sinh \left(x_{0}\right)$, and suppose that $\psi$ satisfies a Dirichlet (resp. Neumann) boundary condition on the slit: For $0 \leq \theta \leq 2 \pi$ we have

$$
\psi(0, \theta) \equiv 0 \quad\left(\text { resp. } \quad \partial_{x} \psi(0, \theta) \equiv 0\right)
$$

Then for $x \geq 0$,

$$
\psi(x, \theta)=\sum_{i} u_{i}(x) \cdot v_{i}(\theta)
$$

where

$$
h=t \cdot \sqrt{E}
$$

and where $u_{i}:\left[0, x_{0}\right) \rightarrow \mathbb{R}$ is a solution to

$$
-u^{\prime \prime}(x)+\left(b_{i}(h)-h^{2} \cosh ^{2}(x)\right) \cdot u(x)=0
$$

with $u(0)=0\left(\right.$ resp. $\left.u^{\prime}(0)=0\right)$.

A solution $u$ to (20) will be called a radial Mathieu function.

In the sequel, our methods will rely upon estimates of the distribution of the $L^{2}$ and $H^{1}$ mass of an eigenfunction on the ellipse. The following lemma will allow us to reduce such estimates to estimates of radial Mathieu functions.

Lemma 5.2. We have

$$
\begin{aligned}
& \int_{S^{1}}|\psi|^{2} d \theta=\sum_{i=0}^{\infty}\left|u_{i}(x)\right|^{2} \\
& \int_{S^{1}}\left|\partial_{\theta} \psi\right|^{2} d \theta+h^{2} \int_{S^{1}}|\psi|^{2} \cos ^{2}(\theta) d \theta=\sum_{i=0}^{\infty} b_{i}(h) \cdot\left|u_{i}(x)\right|^{2} .
\end{aligned}
$$


Proof. By standard Sturm-Liouville theory, the angular Mathieu functions $\left\{v_{i}\right\}$ form a complete and orthogonal set in $L^{2}\left(S^{1}, d \theta\right)$. (See Appendix A) Thus, (21) follows from (19).

From (19), we have $\partial_{\theta} \psi=\sum u_{i} \cdot \partial_{\theta} v_{i}$. Since $v_{i}$ is an eigenfunction with eigenvalue $b_{i}=b_{i}(h)$ we have

$$
-\partial_{\theta}^{2} v_{i}+h^{2} \cos ^{2}(\theta) \cdot v_{i}=b_{i} \cdot v_{i}
$$

By multiplying by $v_{j}$ and integrating by parts, we have

$$
\int_{S^{1}} \partial_{\theta} v_{i} \cdot \partial_{\theta} v_{j} d \theta+h^{2} \int_{S^{1}} v_{i} \cdot v_{j} \cos ^{2}(\theta) d \theta=b_{i} \int_{S^{1}} v_{i} \cdot v_{j} d \theta
$$

Equation (22) then follows from the fact that $\left\{v_{i}\right\}$ is complete and orthogonal.

5.2. Radial convexity estimates. Our estimates of the distribution of the $L^{2}$ mass of a radial Mathieu function $u$ depend upon the following observation. We have

$$
\left(u^{2}\right)^{\prime \prime}=2 \cdot u \cdot u^{\prime \prime}+2 \cdot\left(u^{\prime}\right)^{2} \geq 2 \cdot u \cdot u^{\prime \prime},
$$

and hence if $b_{i}-h^{2} \cosh ^{2}(x) \geq \frac{1}{2}$, then by (20) we have

$$
\left(u^{2}\right)^{\prime \prime} \geq u^{2} \text {. }
$$

We will let $w:[0, X] \rightarrow \mathbb{R}^{+}$denote a smooth function such that

$$
\begin{aligned}
& w^{\prime \prime}(x) \geq w(x), \\
& w(x) \geq 0
\end{aligned}
$$

for all $x \in[0, X]$ and

$$
w^{\prime}(0) \geq 0 .
$$

In particular, if $X$ satisfies

$$
b_{i}-h^{2} \cdot \cosh ^{2}(X) \geq \frac{1}{2},
$$

then the square, $u_{i}^{2}$, of a radial Mathieu function satifying either Dirichlet or Neumann conditions is an example of such a function $w$.

The following expression of convexity is the basis for our estimates.

Lemma 5.3. For all $x, y \geq 0$ such that $x+y \leq X$, we have

$$
w(x+y) \geq w(x) \cdot \cosh (y) .
$$

Proof. The claim holds if $w(x)=0$. So we may assume that $w(x)>0$. Let $z(y)=$ $w(x+y) / w(x)$. Note that $z(0)=1$ and $z^{\prime}(y) \geq 0$. Since $w^{\prime \prime}(x+y) \geq w(x+y)$, we have $\frac{d^{2}}{d y^{2}}(z(y)-\cosh (y)) \geq 0$ and since $w^{\prime}(x) \geq 0$, we have $\left.\frac{d}{d y}\right|_{y=0}(z(y)-\cosh (y)) \geq$ 0 . It follows that $\partial_{y}(z(y)-\cosh (y)) \geq 0$ for all $y \geq 0$. Since $z(0)-\cosh (0)=0$, we have $z(y)-\cosh (y) \geq 0$ for all $y$, and the result follows.

Proposition 5.4. Let $p:[0, X] \rightarrow \mathbb{R}^{+}$be a decreasing integrable function. Then

$$
\int_{0}^{X} p(x) \cdot w(x) d x \leq\left(\frac{p(0)}{\cosh (X / 2)}+p(X / 2)\right) \int_{0}^{X} w(x) d x
$$


Proof. Applying Lemma 5.3 with $y=X / 2$ gives

$$
\cosh (X / 2) \int_{0}^{X / 2} w(x) d x=\int_{0}^{X / 2} w(x+X / 2) d x \leq \int_{X / 2}^{X} w(x) d y
$$

and hence

$$
\int_{0}^{X / 2} w(x) d x \leq \frac{1}{\cosh (X / 2)} \int_{X / 2}^{X} w(x) d y .
$$

Since $p$ is decreasing,

$$
\int_{0}^{X} p(x) \cdot w(x) d x \leq p(0) \int_{0}^{X / 2} w(x) d x+p(X / 2) \int_{X / 2}^{X} w(x) d x .
$$

Combining this with (24) gives the claim.

Proposition 5.5. Let $p:[0, X] \rightarrow \mathbb{R}^{+}$be an increasing integrable function. Then

$$
\int_{0}^{X} w(x) d x \leq \frac{2}{p(X / 2)} \int_{0}^{X} w(x) \cdot p(x) d x .
$$

Proof. From (24) we have

$$
\int_{0}^{X} w(x) d x \leq\left(\frac{1}{\cosh (X / 2)}+1\right) \int_{X / 2}^{X} w(x) d x .
$$

Since $p$ is increasing, we have

$$
\int_{X / 2}^{X} w(x) d x \leq \frac{1}{p(X / 2)} \int_{X / 2}^{X} w(x) \cdot p(x) d x .
$$

By combining these inequalities and using the fact that $\cosh (X / 2) \geq 1$, we obtain the claim.

\section{LIMITS FOR ANALYTIC EIGENBRANCHES}

In this section we prove that each real-analytic eigenvalue branch $E_{t}$ converges. The proof consists of three main steps. First we prove that $t^{2} \cdot E_{t}$ converges as $t$ tends to zero. We use this to then prove that $t^{2 k} E_{t}$ converges for some $k<1$. Finally, we use this to prove that $E_{t}$ converges. At each new stage, the previous estimate is used to control $h(t)$ along the eigenbranch.

\subsection{Convergence relative to $t^{2}$.}

Proposition 6.1. Let $E_{t}$ be any eigenbranch. Then

$$
\lim _{t \rightarrow 0^{+}} t^{2} \cdot E_{t}
$$

exists and is finite.

Proof. Since $\cosh ^{2}(x) \geq 1$, by comparing (13) and (17) we find that

$$
t \cdot \dot{q}_{U}(\psi) \geq-q(\psi)
$$

and by comparing (14) and (18),

$$
t \cdot \dot{N}_{U}(\psi) \leq N(\psi)
$$


Hence by Corollary 4.11 we have

$$
\dot{E} \geq-\left(C+\frac{2}{t}\right) E
$$

for some constant $C$. Lemma 4.8 then allows us to conclude the proof since in this case $F(t)=C t^{2} E_{t}$ for some constant $C$.

6.2. Convergence relative to $t^{2 k}$. By Proposition 6.1 the parameter $h=t \cdot \sqrt{E}$ in the radial Mathieu equation associated to $\psi$ is uniformly bounded in $t$. This will allow us to prove the following.

Theorem 6.2. Let $E_{t}$ be any eigenbranch. Then there exists $k<1$ such that

$$
\lim _{t \rightarrow 0^{+}} t^{2 k} \cdot E_{t}
$$

exists and is finite.

Proof. It suffices to show that there exists $\kappa<1$ so that

$$
t \cdot \dot{N}_{U}(\psi) \leq \kappa \cdot N(\psi) .
$$

Then we could argue as in the proof of Proposition 6.1 where the ' 2 ' that appears in (25) is replaced by $\kappa+1$. In particular, the desired $k$ equals $(\kappa+1) / 2$.

To verify (26), we will use the following.

Lemma 6.3. There exists $M>0$ such that

$$
\int_{U_{t}}|\psi|^{2} d x d \theta \leq M \int_{U_{t}}|\psi|^{2} \sinh ^{2} x d x d \theta
$$

Assuming the lemma, we finish the proof of the theorem. Let $0<\kappa<1$ be such that $M=\kappa /(1-\kappa)$. Then

$$
(1-\kappa) \int_{U_{t}}|\psi|^{2} \sin ^{2} \theta d x d \theta \leq(1-\kappa) \int_{U_{t}}|\psi|^{2} d x d \theta \leq \kappa \int_{U_{t}}|\psi|^{2} \sinh ^{2} x d x d \theta
$$

and hence

$$
\int_{U_{t}}|\psi|^{2} \sin ^{2} \theta d x d \theta \leq \kappa \int_{U_{t}}|\psi|^{2} \sinh ^{2} x d x d \theta+\kappa \int_{U_{t}}|\psi|^{2} \sin ^{2} \theta d x d \theta .
$$

Estimate (26) follows then by comparing (14) and (18).

Proof of Lemma 6.3. By Lemma 5.2. it suffices to prove that there exists $M$ so that for all $i$,

$$
\int_{0}^{Y_{t}}\left|u_{i}\right|^{2} d x \leq M \int_{0}^{Y_{t}}\left|u_{i}\right|^{2} \sinh ^{2} x d x
$$

Let $p(x)=\sinh ^{2}(x)$, and let $X=\sinh ^{-1}(1)$. Since $p$ is increasing on $[0, \infty)$, the infimum of $p(x)$ over $[X, \infty)$ equals $p(X)=1$. In particular, if $t \leq r_{0}$, then $Y_{t} \geq X$, and we obtain

$$
\int_{X}^{Y_{t}}\left|u_{i}\right|^{2} p(x) d x \geq \int_{X}^{Y_{t}}\left|u_{i}\right|^{2} d x
$$

By Proposition 6.1, $h=h(t)$ is uniformly bounded. Choose $i_{0} \in \mathbb{Z}^{+}$so that $i_{0}^{2} \geq 2 h^{2}(t)+1 / 2$ for all sufficiently small $t$. Then since $b_{i}(t) \geq i^{2}$ (see Appendix A) and $\cosh ^{2}(X)=2$, we have that for all $x \in[0, X]$ and $i \geq i_{0}$,

$$
b_{i}(t)-h(t)^{2} \cdot \cosh ^{2}(x) \geq \frac{1}{2} .
$$


Since $\psi$ satisfies either Neumann or Dirichlet conditions along the slit, we have either $u_{i}^{\prime}(0)=0$ or $u_{i}(0)=0$ for all $i$. Note also that $p$ is increasing.

Thus, for $i \geq i_{0}$, we may apply Proposition [5.5 with $w=u_{i}^{2}$ to find that

$$
\int_{0}^{X}\left|u_{i}\right|^{2} d x \leq \frac{2}{p(X / 2)} \int_{0}^{X}\left|u_{i}\right|^{2} p(x) d x .
$$

The lemma is thus proved for $i \geq i_{0}$ large enough.

For $i<i_{0}$, we note that by Proposition 6.1, $h$ is bounded, and hence $b_{i}$ is bounded. Thus, the claim for $i<i_{0}$ follows from the fact that the solution of the ordinary differential equation (20) with a fixed boundary condition depends continuously on parameters.

In the next section we make crucial use of the following variant of Theorem 6.2.

Corollary 6.4. Let $h(t)=t \cdot \sqrt{E_{t}}$. There exists $\epsilon_{0}>0$ so that

$$
\lim _{t \rightarrow 0} t^{-\epsilon_{0}} h(t)=0 \text {. }
$$

\subsection{Convergence of $E_{t}$.}

Theorem 6.5. Let $E_{t}$ be a real-analytic eigenbranch. Then

$$
\lim _{t \rightarrow 0^{+}} E_{t}
$$

exists and is finite.

Proof. It suffices to show that there exists $\delta>0$ so that for all sufficiently small $t$,

$$
\dot{q}_{U}(\psi) \geq-t^{\delta-1} \cdot q_{U}(\psi)-2 t^{\delta-1} \cdot E \cdot N_{U}(\psi)
$$

and

$$
\dot{N}_{U}(\psi) \leq t^{\delta-1} \cdot N_{U}(\psi)
$$

For then by Corollary 4.11, there would exist $C>0$ so that

$$
\dot{E} \geq-\left(C+4 \cdot t^{\delta-1}\right) E .
$$

Since $t^{\delta-1}$ is integrable near $t=0$, the claim would then follow from Lemma 4.8,

By comparing (13) to (15) and (14) to (18), we see that to prove (29) and (30) it suffices to prove that there exists $\delta>0$ so that for sufficiently small $t$ we have

$$
\int_{U_{t}} \frac{\left|\partial_{\theta} \psi_{t}\right|^{2}}{\cosh ^{2} x} d x d \theta \leq t^{\delta}\left(\int_{U_{t}}\left|\partial_{\theta} \psi_{t}\right|^{2} d x d \theta+2 t^{2} \cdot E \cdot \int_{U_{t}} \psi^{2} \sinh ^{2} x d x d \theta\right)
$$

and

$$
\int_{U_{t}}\left|\psi_{t}\right|^{2} d x d \theta \leq t^{\delta} \int_{U_{t}}\left|\psi_{t}\right|^{2} \sinh ^{2} x d x d \theta
$$

These estimates can be further reduced to estimates of Mathieu functions as in \$5.2. In order to state these estimates, we first note that, by Corollary 6.4 there exists $\epsilon>0$ such that

$$
\lim _{t \rightarrow 0} t^{-2 \epsilon} \cdot h(t)=0 .
$$

We fix this $\epsilon$ in what follows. 
Lemma 6.6. Let $u_{i}$ be a solution to the radial Mathieu equation (20) with $h=h(t)$ satisfying (34). There exists $t_{0}$ so that if $t<t_{0}$, then for all $i \geq 0$,

$$
\int_{0}^{Y_{t}}\left|u_{i}\right|^{2} d x \leq t^{\epsilon / 2} \int_{0}^{Y_{t}}\left|u_{i}\right|^{2} \sinh ^{2} x d x
$$

and for $i>0$,

$$
\int_{0}^{Y_{t}} \frac{\left|u_{i}\right|^{2}}{\cosh ^{2} x} d x \leq t^{\epsilon / 2} \int_{0}^{Y_{t}}\left|u_{i}\right|^{2} d x
$$

Assuming this lemma, we finish the proof of Theorem 6.5. Using (21) we see that (33) follows immediately from (35) with $\delta=\epsilon / 2$.

To verify (32) we use (22) to find that

$$
\int_{U_{t}} \frac{\left|\partial_{\theta} \psi\right|^{2}}{\cosh ^{2}(x)} d x d \theta \leq \sum_{i=0}^{\infty} b_{i} \int_{0}^{Y_{t}} \frac{u_{i}^{2}(x)}{\cosh ^{2}(x)} d x .
$$

In Appendix $\mathrm{A}$ we show that $b_{0} \sim \frac{1}{2} h^{2}$ for small $h$. Hence since $h^{2}=t^{2} E$, we find that for small $t$,

$$
\begin{aligned}
b_{0} \int_{0}^{Y_{t}} \frac{u_{0}^{2}(x)}{\cosh ^{2}(x)} d x & \leq h^{2} \int_{0}^{Y_{t}} u_{0}^{2}(x) d x \\
& \leq E \cdot t^{2} \cdot t^{\epsilon / 2} \int_{0}^{Y_{t}} u_{0}^{2}(x) \sinh ^{2} x d x \\
& \leq t^{\epsilon / 2} \cdot t^{2} \cdot E \int_{U_{t}}|\psi|^{2} \sinh ^{2} x d x d \theta .
\end{aligned}
$$

Here we have used (35). Using (22) and (36), we find that

$$
\begin{aligned}
\sum_{i=1}^{\infty} b_{i} \int_{0}^{Y_{t}} \frac{u_{i}^{2}(x)}{\cosh ^{2}(x)} d x & \leq t^{\delta} \sum_{i=1}^{\infty} b_{i} \int_{0}^{Y_{t}} u_{i}^{2}(x) d x \\
& \leq t^{\delta}\left(\int_{U_{t}}\left|\partial_{\theta} \psi\right|^{2} d x d \theta+h^{2} \int_{U_{t}}|\psi|^{2} d x d \theta\right) .
\end{aligned}
$$

Thus, since $h^{2}=t^{2} E$, and using (36) to estimate the last integral of the previous inequality, equation (32) follows with $\delta=\epsilon / 2$.

6.4. The individual estimates. The remainder of this section is dedicated to proving Lemma 6.6. The behavior of the radial Mathieu function $u_{i}$ for small $h$ is qualitatively different if $i=0$ than if $i \neq 0$. We prove the cases $i \neq 0$ with Lemma 6.7 and the case $i=0$ with Lemma 6.9.

6.4.1. Estimates for $i \neq 0$.

Lemma 6.7. There exists some $t_{0}$ and some constants $C_{1}, C_{2}$ such that for any $t<t_{0}$ and for any $i \neq 0$, we have

$$
\int_{0}^{Y_{t}} \frac{u_{i}^{2}(x)}{\cosh ^{2}(x)} d x \leq C_{1} \cdot t^{\epsilon} \int_{0}^{Y_{t}} u_{i}^{2}(x) d x
$$

and

$$
\int_{0}^{Y_{t}} u_{i}^{2}(x) d x \leq C_{2} \cdot t^{\epsilon} \int_{0}^{Y_{t}} u_{i}^{2}(x) \sinh ^{2}(x) d x
$$


Proof. Define $X_{t} \in[0, \infty)$ implicitly by $\cosh \left(X_{t}\right)=t^{-\epsilon}$. Then we have

$$
\int_{X_{t}}^{Y_{t}} \frac{u_{i}^{2}(x)}{\cosh ^{2}(x)} d x \leq t^{2 \epsilon} \int_{X_{t}}^{Y_{t}} u_{i}^{2}(x) d x .
$$

Since $t^{2 \epsilon}=o\left(t^{\epsilon}\right)$, it suffices to estimate the integral over $\left[0, X_{t}\right]$.

By (34), we have that $h(t)^{2} \cdot \cosh \left(X_{t}\right)^{2}$ tends to zero as $t$ tends to zero. Since $i \neq 0$, we also have $b_{i} \geq 1$. (See Appendix $\mathrm{A}$ ) Thus, for sufficiently small $t$,

$$
b_{i}(h(t))-h(t)^{2} \cdot \cosh ^{2}\left(X_{t}\right) \geq \frac{1}{2} .
$$

Therefore, we can apply the estimates of $\$ 5.2$ with $w=u^{2}$ and $X=X_{t}$. By applying Proposition 5.4 with $p=\cosh ^{-2}$, we find that

$$
\int_{0}^{X_{t}} \frac{u_{i}^{2}(x)}{\cosh ^{2}(x)} d x \leq \frac{2}{\cosh \left(X_{t} / 2\right)} \int_{0}^{X_{t}} u_{i}^{2}(x) d x .
$$

Since $\cosh \left(X_{t} / 2\right)=\sqrt{\left(\cosh \left(X_{t}\right)+1\right) / 2}=\sqrt{\left(t^{-\epsilon}+1\right) / 2}$, we have

$$
\int_{0}^{X_{t}} \frac{u_{i}^{2}(x)}{\cosh ^{2}(x)} d x \leq \frac{2 \sqrt{2} \cdot t^{\epsilon}}{1+t^{\epsilon}} \int_{0}^{X_{t}} u_{i}^{2}(x) d x .
$$

Estimate (37) then follows for $C_{1}=2 \sqrt{2}$ and any $t$ small enough so that $t^{2 \epsilon} \leq C_{1} \cdot t^{\epsilon}$.

The proof of the estimate (38) is similar. From the definition of $X_{t}$ we have

$$
\int_{X_{t}}^{Y_{t}} u_{i}^{2} d x \leq \frac{2 t^{2 \epsilon}}{1-t^{2 \epsilon}} \int_{X_{t}}^{Y_{t}} u_{i}^{2} \cdot \sinh (x)^{2} d x
$$

and from Corollary $[5.4$ we find that

$$
\int_{0}^{X_{t}} u_{i}^{2}(x) d x \leq \frac{8 t^{\epsilon}}{1-t^{\epsilon}} \int_{0}^{X_{t}} \sinh ^{2}(x) \cdot u_{i}^{2}(x) d x ;
$$

the estimate follows with any $C_{2}>8$ and $t$ small enough so that $\frac{2 t^{2 \epsilon}}{1-t^{2 \epsilon}}$ and $\frac{8}{1-t^{\epsilon}}$ are less than $C_{2}$.

Since equation (37) implies equation (36), and equation (38) implies equation (35), the estimates of Lemma 6.6 are proven for any index $i>0$. It remains to prove (35) for $i=0$.

6.4.2. The estimate for $i=0$. Since $b_{0}(h)$ is real-analytic in $h^{2}$, we can write

$$
b_{0}(h)=a(h) \cdot h^{2}
$$

for some analytic function $a$. It follows from Theorem 6.2 that $a(h(t))$ is bounded near $t=0$. The Mathieu equation for $i=0$ can thus be rewritten as

$$
u_{0}^{\prime \prime}(x)=h(t)^{2} \cdot\left(a(h(t))-\cosh ^{2}(x)\right) \cdot u_{0}(x) .
$$

We will treat this equation as a perturbation of $u_{0}^{\prime \prime}=0$. To make this precise, we set $t_{0}>0$ and define

$$
M(X)=\sup \left\{\left|a(h(t))-\cosh ^{2}(x)\right| \mid 0 \leq x \leq X \text { and } 0 \leq t \leq t_{0}\right\} .
$$


Lemma 6.8. Given a solution u to (39) define $R$ by

$$
R(x)=u(x)-\left(u(0)+u^{\prime}(0) \cdot x\right) .
$$

Then for all $t \in\left(0, t_{0}\right)$, and for any positive weight $p$, the function $R$ satisfies

$$
\int_{0}^{X}|R(x)|^{2} p(x) d x \leq h(t)^{4} \cdot M(X)^{2} \cdot X^{4} \cdot L(X) \cdot \int_{0}^{X}|u(x)|^{2} d x,
$$

where

$$
L(X)=\sup _{x \in[0, X]} p(x) .
$$

Proof. By applying the method of variation of constants (or, equivalently, Duhamel's principle) we find that

$$
R(x)=h(t)^{2} \int_{0}^{x}(x-y)\left(a(h(t))-\cosh ^{2}(y)\right) u(y) d y .
$$

Thus, for $t<t_{0}$ and $0<x<X$,

$$
|R(x)|^{2} \leq h^{4} \cdot M(X)^{2}\left(\int_{0}^{x}(x-y) \cdot|u(y)| d y\right)^{2} .
$$

By applying the Bunyakovsky-Cauchy-Schwarz inequality we find that

$$
\begin{aligned}
|R(x)|^{2} & \leq h^{4} \cdot M^{2}(X)\left|\int_{0}^{x}(x-y)^{2} d y\right| \cdot\left|\int_{0}^{x} u(y)^{2} d y\right| \\
& \leq h^{4} \cdot M^{2}(X) \cdot\left(|x|^{3} / 3\right) \cdot\left|\int_{0}^{x} u(y)^{2} d y\right|
\end{aligned}
$$

for all $|x| \leq X$. Thus,

$$
\int_{0}^{X}|R(x)|^{2} p(x) d x \leq h^{4} \cdot M^{2}(X) \cdot X^{3} \cdot L(X) \int_{0}^{X}\left|\int_{0}^{x} u(y)^{2} d y\right| d x .
$$

The desired estimate follows.

The following completes the proof of Lemma 6.6.

Lemma 6.9. Let $\epsilon>0$ be as in (34). There exists some constant $C$ such that, if we let $u=u_{0}$ be a solution to the radial Mathieu equation (20) associated to $b_{0}(t)$ and $h(t)$, and if $u$ satisfies either $u(0)=0$ or $u^{\prime}(0)=0$, then for any sufficiently small t we have

$$
\int_{0}^{Y_{t}} u^{2} \leq C \cdot t^{\epsilon} \int_{0}^{Y_{t}} u^{2}(x) \sinh ^{2}(x) d x
$$

Proof. As above, let $X_{t} \geq 0$ be defined by $\cosh \left(X_{t}\right)=t^{-\epsilon}$. Let $a=u^{\prime}(0)$ and $b=u(0)$. Since $u(x)^{2} \leq 2(a x+b)^{2}+2 R(x)^{2}$, by applying Lemma 6.8 with $p(x) \equiv 1$, we find that

$$
\int_{0}^{X_{t}} u^{2}(x) d x \leq 2 \int_{0}^{X_{t}}(a x+b)^{2} d x+K(t) \int_{0}^{X_{t}} u^{2}(x) d x,
$$

where

$$
K(t)=2 M^{2}\left(X_{t}\right) \cdot h(t)^{4} \cdot X_{t}^{4} .
$$

In other words,

$$
\int_{0}^{X_{t}} u^{2}(x) d x \leq \frac{2}{1-K(t)} \int_{0}^{X_{t}}(a x+b)^{2} d x,
$$


provided that $K(t)$ is less than 1 . As $t$ tends to zero, $X_{t} \sim \epsilon|\ln t|$ tends to infinity, and hence $M\left(X_{t}\right) \sim \cosh ^{2}\left(X_{t}\right) \sim t^{-2 \epsilon}$. It follows that $K(t) \sim\left(t^{-\epsilon} \cdot h \cdot \epsilon|\ln h|\right)^{4}$. Thus, by (34), $K(t)$ tends to zero as $t$ tends to zero. Therefore, for $t$ sufficiently small,

$$
\int_{0}^{X_{t}} u^{2}(x) d x \leq 4 \int_{0}^{X_{t}}(a x+b)^{2} d x .
$$

If $u(0)=0$, then $a x+b=u^{\prime}(0) \cdot x$, and if $u^{\prime}(0)=0$, then $a x+b=u(0)$. A straightforward calculation gives a constant $C$ such that for all sufficiently large $X$, we have

$$
\int_{0}^{X} 1 d x \leq \frac{C \cdot X}{\sinh ^{2}(X)} \int_{0}^{X} \sinh ^{2}(x) d x
$$

and

$$
\int_{0}^{X} x^{2} d x \leq \frac{C \cdot X}{\sinh ^{2}(X)} \int_{0}^{X} x^{2} \sinh ^{2}(x) d x .
$$

Thus, since $\lim _{t \rightarrow 0} X_{t}=\infty$, for small $t$ we have

$$
\int_{0}^{X_{t}}(a x+b)^{2} d x \leq \frac{C \cdot X_{t}}{\sinh ^{2}\left(X_{t}\right)} \int_{0}^{X}(a x+b)^{2} \sinh ^{2}(x) d x .
$$

By applying Lemma 6.8 with $p(x)=\sinh ^{2}(x)$, we obtain

$$
\int_{0}^{X_{t}}(a x+b)^{2} \sinh ^{2}(x) d x \leq 2 \int_{0}^{X_{t}} u(x)^{2} \sinh ^{2}(x) d x+\tilde{K}(t) \int_{0}^{X_{t}} u(x)^{2} d x,
$$

where $\tilde{K}(t)=K(t) \cdot \sinh ^{2}\left(X_{t}\right)$. By combining this with (42) and (43) we have

$$
\int_{0}^{X_{t}} u^{2}(x) d x \leq \frac{8 C \cdot X_{t}}{\sinh ^{2}\left(X_{t}\right)} \int_{0}^{X_{t}} u^{2}(x) \sinh ^{2}(x) d x+4 C \cdot X_{t} \cdot K(t) \int_{0}^{X_{t}} u^{2}(x) d x .
$$

Arguing as above, one sees that $X_{t} \cdot K(t)$ tends to zero as $t$ tends to zero. It follows that for all sufficiently small $t$, we have

$$
\int_{0}^{X_{t}} u^{2} d x \leq \frac{9 C \cdot X_{t}}{\sinh ^{2}\left(X_{t}\right)} \int_{0}^{X_{t}} u^{2}(x) \sinh ^{2}(x) d x .
$$

Note that $C X_{t} / \sinh ^{2}\left(X_{t}\right) \leq t^{\epsilon}$ for sufficiently small $t$.

Since

$$
\inf _{\left[X_{t}, Y_{t}\right]} \sinh ^{2}(x)=\sinh ^{2}\left(X_{t}\right)=t^{-2 \epsilon}-1
$$

we also have

$$
\int_{X_{t}}^{Y_{t}} u^{2}(x) d x \leq \frac{t^{2 \epsilon}}{1-t^{2 \epsilon}} \int_{X_{t}}^{Y_{t}} u^{2}(x) \sinh ^{2}(x) d x
$$

The claim follows.

\section{Generic Simplicity FOR POLYGONS}

In this section we combine the convergence of analytic eigenvalue branches with the convergence of eigenfunctions to generalize our earlier results HlrJdg09 on the spectral simplicity of simply connected polygons to several settings. 
7.1. Slits and simplicity. Our results on spectral simplicity for polygons depend on the following.

Proposition 7.1. Let $\Sigma_{t}$ be a slit of length $2 t$ centered at a point $p$ belonging to the interior of a Lipschitz domain $\Omega \subset \mathbb{R}^{2}$. Let $D$ be a measurable subset of $\partial \Omega$ and let $D_{t}^{\prime} \subset \Omega_{\Sigma}$ be either $D$ or $D \cup \Sigma$. If the spectrum of $q$ on $H_{D}^{1}(\Omega)$ is simple, then for all but countably many $t$, the spectrum of $q$ on $H_{D_{t}^{\prime}}^{1}\left(\Omega_{\Sigma_{t}}\right)$ is simple.

Proof. By Theorem 4.2, the eigenvalues vary analytically for $t>0$. Hence it suffices to show that there does not exist a real-analytic eigenvalue branch $E_{t}$ such that the dimension of the associated eigenspace $V_{t}$ is greater than 1 for each $t>0$.

Suppose that $\psi_{t}$ and $\psi_{t}^{*} \in V_{t}$ denote normalized real-analytic eigenfunction branches that are mutually orthogonal for each $t$. By Theorem 6.5. the corresponding eigenvalue branch converges to some $E_{0}$ when $t$ goes to 0 . By Theorem 3.1. $\psi_{t}$ and $\psi_{t}^{*}$ converge to eigenfunctions $\psi$ and $\psi^{*}$ on $\Omega_{\Sigma}$ with the same eigenvalue $E_{0}$. Since $\psi_{t}$ and $\psi_{t}^{*}$ are normalized and orthogonal for each $t$, the limits $\psi$ and $\psi^{*}$ are orthogonal. But this contradicts the assumption that the spectrum of $\Omega_{\Sigma}$ is simple.

Remark 7.2. The proof applies equally well if $\Omega$ is a slit domain with simple spectrum. Thus one can iterate the procedure, and prove that for all but countably many choices of slit lengths, the domain $\Omega$ slit along finitely many slits has a simple spectrum. Actually, if we take all the slits of the same length $t$, the spectrum is also simple for all but countably many choices of $t$, but to prove this result one has to adapt Theorem 6.5 to the case of several slits. This is easily done using the localization principle and the estimates we have proved.

By combining Theorem 7.1, Remark 7.2, and the main result of [HlrJdg09], we obtain the simplicity of the spectrum of the generic simply connected slit polygon. To be precise, let $\mathcal{S}_{n, k}$ be the set of simply connected $n$-gons $P$ with $k$ disjoint slits $\Sigma_{1}, \Sigma_{2}, \ldots, \Sigma_{k}$. Note that the vertices of $P$ and the endpoints of each slit $\Sigma_{1}, \Sigma_{2}, \ldots, \Sigma_{k}$, determine the slit polygon $P_{\Sigma_{1} \cup \Sigma_{2} \cup \cdots \cup \Sigma_{k}}$. Thus, $\mathcal{S}_{n, k}$ may be naturally identified with an open subset of $\mathbb{R}^{2 n+2 k+2 k}$. In particular, $\mathcal{S}_{n, k}$ inherits a natural affine structure and Borel measure.

Theorem 7.3. If $n \geq 4$ and $k \geq 0$, then almost every slit polygon in $\mathcal{S}_{n, k}$ has simple Dirichlet or Neumann spectrum.

Proof. Let $P$ be a simply connected $n$-gon. Let $C=\left\{c_{1}, \ldots, c_{k}\right\}$ be a set of $k$ distinct points in $P$, and let $L=\left\{\ell_{1}, \ldots, \ell_{k}\right\}$ be a set of (not necessarily distinct) lines that pass through the origin in $\mathbb{R}^{2}$. Let $A(P, C, L)$ be the set of slit polygons $P_{\Sigma_{1} \cup \Sigma_{2} \cup \cdots \cup \Sigma_{k}}$, where $\Sigma_{i}$ is centered at $c_{i}$ and is parallel to $\ell_{i}$. Note that a point in $A(P, C, L)$ is determined by the lengths of the slits.

The sets $A(P, C, L)$ provide a natural smooth foliation of $\mathcal{S}_{n, k}$ by $k$-dimensional planar sets. By Proposition 7.1 and Remark 7.2, if $P$ has a simple spectrum, then almost every slit polygon in $A(P, C, L)$ has a simple spectrum. By the main result of HlrJdg09, almost every simply connected $n$-gon has a simple spectrum. The result follows by integrating transversely to this foliation using Fubini's theorem.

7.2. Polygons with mixed boundary conditions. Let $P$ be a simply connected polygon, and let $D$ be a union of a set of edges in the boundary, $\partial P$, of $P$. Recall from $\sqrt{2}$ that the eigenfunctions of the Dirichlet energy $q$ on $H_{D}^{1}(P)$ satisfy Dirichlet 
conditions on $D$ and Neumann conditions on $\partial P \backslash D$. We say that $(P, D)$ has a simple spectrum if and only if the quadratic form $q$ on $H_{D}^{1}(P)$ has a simple spectrum.

Let $v_{1}, v_{2}, \ldots, v_{n}$ be a cyclic ordering of the edges of a simply connected polygon $P$. Let $e_{i}$ denote the boundary edge joining the vertex $v_{i}$ to $v_{i+1} 4$ Given a subset $\sigma \subset\{1,2, \ldots, n\}$, let $\mathcal{P}_{n, \sigma}$ denote the set of pairs $(P, D)$, where $P$ is a simply connected $n$-gon 5 and

$$
D=\bigcup_{i \in \sigma} e_{i} .
$$

The set $\mathcal{P}_{n, \sigma}$ can be naturally identified with an open subset of $\mathbb{R}^{2 n}$ and hence has a natural Borel measure and affine structure.

Theorem 7.4. If $n \geq 4$, then almost every polygon in $\mathcal{P}_{n, \sigma}$ has a simple spectrum.

Proof. By arguing as in HlrJdg09], we see that it suffices to construct one polygon in $\mathcal{P}_{n, \sigma}$ that has a simple spectrum.

We first construct such a polygon in the case of alternating boundary conditions; that is, we suppose that $i \in \sigma$ if and only if $i+1 \notin \sigma$, where as usual $n+1 \equiv 1$. Note that $n$ is even in this case.

Let $Q$ be the rectangle $[0, a] \times[0,1]$, and let $D=\{0,1\} \times[0, a]$. That is, we consider the eigenvalue problem on $Q$ with Dirichlet conditions on the vertical edges and Neumann conditions on the horizontal edges. The set of functions of the form

$$
\sin \left(\frac{m_{1} \pi}{a} \cdot x\right) \cdot \cos \left(m_{2} \pi \cdot y\right)
$$

where $m_{1}>0$ and $m_{2} \geq 0$ are integers, is an orthonornal basis of eigenfunctions. Thus, the set of eigenvalues is $\left\{\left(m_{1} \pi / a\right)^{2}+\left(m_{2} \pi\right)^{2}\right\}$. In particular, $(Q, D)$ has a simple spectrum if and only if $a^{2} \notin \mathbb{Q}$.

Let $c_{1}, c_{2}, \ldots, c_{k}$ be $k=n / 2-2$ distinct points on the horizontal segment $[0, a] \times$ $\{1 / 2\}$. By Theorem 7.1 there exist horizontal slits $\Sigma_{1}, \ldots, \Sigma_{k}$ centered respectively at $c_{i}$ such that $\left(Q_{\Sigma_{1} \cup \cdots \cup \Sigma_{k}}, D^{\prime}\right)$ has a simple spectrum, where $D^{\prime}=D \cup \Sigma_{1} \cup \cdots \cup \Sigma_{k}$. See Figure 1 .

The slit domain $Q_{\Sigma_{1} \cup \ldots \cup \Sigma_{k}}$ has a reflection symmetry $\tau$ induced by $(x, y) \mapsto$ $(x, 1-y)$. A standard argument shows that restricting functions on $Q_{\Sigma_{1} \cup \ldots \cup \Sigma_{k}}$ to the rectangle $Q^{\prime}=[0, a] \times[0,1 / 2]$ defines a bijection between $\tau^{*}$-invariant eigenfunctions of $q$ on $H^{1}\left(Q_{\Sigma_{1} \cup \ldots \cup \Sigma_{k}}, D^{\prime}\right)$ and the eigenfunctions of $q$ on $H_{D^{\prime}}^{1}\left(Q^{\prime}\right)$.

Therefore, since the spectrum of $\left(Q_{\Sigma_{1} \cup \ldots \cup \Sigma_{k}}, D^{\prime}\right)$ is simple, the spectrum of $\left(Q^{\prime}, D^{\prime}\right)$ is also simple. By placing vertices at the endpoints of the slit, we may regard the rectangle $Q^{\prime}$ as a polygon with $n=2 k+4$ vertices. From this viewpoint, the boundary conditions given by $D^{\prime}$ alternate as desired.

Given a general subset $\sigma \subset\{1,2, \ldots, n\}$, let $F$ be the finite set obtained by identifying $i$ and $i+1$ if they either both belong to $\sigma$ or if they both do not belong to $\sigma$. The cyclic ordering of $\{1,2, \ldots, n\}$ induces a cyclic ordering on $F$, and hence we may identify $F$ with $\left\{1,2, \ldots, n^{\prime}\right\}$ for some $n^{\prime} \leq n$. Note that $n^{\prime}$ is either 1 or even. Let $\sigma^{\prime} \subset\left\{1,2, \ldots, n^{\prime}\right\}$ denote the set obtained by identifying $i \in \sigma$ with $i+1$ if it also belongs to $\sigma$.

\footnotetext{
${ }^{4}$ Here $e_{n}$ joins $v_{n}$ and $v_{1}$.

${ }^{5}$ By a simply connected polygon, we mean a compact set whose boundary consists of finitely many line segments and whose interior is simply connected.
} 
$(0,1)$

$(\mathrm{a}, 1)$



Figure 1. The slit rectangle

The set $\sigma^{\prime} \subset\left\{1,2, \ldots, n^{\prime}\right\}$ is alternating, and hence, if $n^{\prime} \geq 4$, there exists an element $\left(P^{\prime}, D\right)$ in $\mathcal{P}_{n^{\prime}, \sigma^{\prime}}$ with a simple spectrum. By judiciously adding vertices to the boundary edges of the $n^{\prime}$-gon $P$, we obtain an $n$-gon $P^{\prime}$ such that the set $D$ corresponds to $\sigma$. Thus $(P, D)$ is an element of $\mathcal{P}_{n, \sigma}$ with a simple spectrum.

If $n^{\prime}=2$, then we consider the eigenvalue problem on $(Q, D)$, where $Q=[0, a] \times$ $[0,1]$ and $D$ is either $\{0\} \times[0,1]$ or its complement. In either case a basis of eigenfunctions can be constructed by taking products of sines and cosines. By making an appropriate choice of $a$, one finds that the spectrum of $(Q, D)$ is simple. By adding vertices appropriately to the boundary edges of $Q$, we obtain $\left(Q^{\prime}, D\right) \in$ $\mathcal{P}_{n, \sigma}$ with a simple spectrum.

The case $n^{\prime}=1$ is the main result of HlrJdg09 and follows from the same construction.

7.3. Multiply connected polygons. We first make precise the definition of a multiply connected polygon. Let $P_{0}, P_{1}, \ldots, P_{k}$ be a finite collection of simply connected polygons. Assume that

(1) $P_{i}$ is contained in the interior of $P_{0}$ for all $i>0$, and

(2) $P_{i} \cap P_{j}=\emptyset$ for all $i, j \geq 0, i \neq j$.

The multiply connected polygon $P$ determined by $P_{0}, P_{1}, \ldots, P_{k}$ is obtained by removing the interiors of the polygons $P_{1}, \ldots, P_{k}$ from the polygon $P_{0}$. In other words, $P$ is the closure of

$$
P_{0} \backslash\left(\bigcup_{i=1}^{k} P_{i}\right) .
$$

Let $\vec{n}=\left(n_{0}, n_{1}, \ldots, n_{k}\right)$ denote a vector of integers with $n_{i} \geq 3$ for each $i$. Let $\mathcal{P}(\vec{n})$ denote the set of all collections of polygons $P_{0}, P_{1}, \ldots, P_{k}$ satisfying (1) and (2) above and such that for each $i$, the polygon $P_{i}$ has $n_{i}$ (ordered) vertices $v_{i, 1}, \ldots, v_{i, n_{i}}$. Since the (ordered) vertices determine the polygon, $\mathcal{P}(\vec{n})$ is naturally in bijective correspondence with an open subset of $\mathbb{R}^{d}$ where $d=2 n_{0}+\cdots+2 n_{k}$. In particular, $\mathcal{P}(\vec{n})$ inherits an affine structure and a Borel measure. 
Proposition 7.5. The space $\mathcal{P}(\vec{n})$ is path connected.

Proof. In HlrJdg09, we proved that the space $\mathcal{P}_{n}$ of simply connected $n$-gons is connected using a construction which we will call 'deleting a vertex'. In particular, given a simply connected $n$-gon $P$, we constructed a linear path $t \mapsto P(t)$ of $n$-gons in $\mathcal{P}_{n}$ with $P(0)=P$ and such that $P(1)$ has three consecutive vertices that belong to the same boundary edge. See Figure 2, We can regard the polygon $P(1)$ as an element of $\mathcal{P}_{n-1}$. Thus, since the space of triangles, $\mathcal{P}_{3}$, is connected, $\mathcal{P}_{n}$ is connected for $n \geq 3$ by induction.

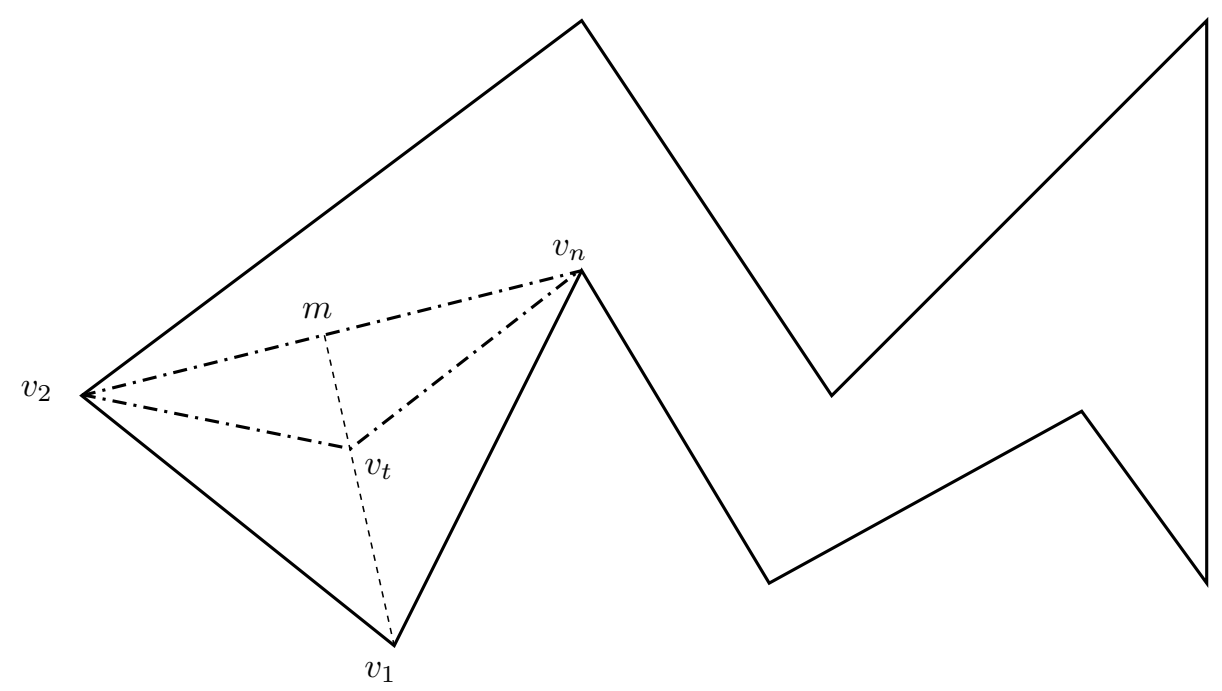

Figure 2. Deleting a vertex

Suppose now that $P \in \mathcal{P}(\vec{n})$ is defined by the simply connected polygons $P_{0}, P_{1}$, $\ldots, P_{k}$, where $n_{i}>3$ for some $i>0$. Let $t \mapsto P_{i}(t)$ denote the path from $P_{i}=P_{i}(0)$ to a polygon $P_{i}(1)$ having three consecutive vertices on the same edge. Let $t \mapsto P(t)$ denote the path of polygons determined by $P_{1}, \ldots, P_{i-1}, P_{i}(t), P_{i+1}, \ldots, P_{k}$. The polygon $P(1)$ may be regarded as an element of $\mathcal{P}\left(n_{0}, \ldots, n_{i-1}, n_{i}-1, n_{i+1}, \ldots, n_{k}\right)$.

Therefore, by induction on the vector $\vec{n}$, we find that it suffices to prove that the space $\mathcal{P}(n, 3,3,3, \ldots, 3)$ is connected. By rescaling the 'interior' triangles if necessary, we may delete vertices of the polygon $P_{0}$ to obtain a path to an element of $\mathcal{P}(3,3,3,3, \ldots, 3)$. An elementary argument then gives that $\mathcal{P}(3,3,3,3, \ldots, 3)$ is connected.

Theorem 7.6. If $n_{0} \geq 4$, then almost every polygon in $\mathcal{P}(\vec{n})$ has a simple spectrum.

Proof. Since $\mathcal{P}(\vec{n})$ is connected, by arguing as in HlrJdg09, we see that it suffices to construct one polygon in $\mathcal{P}(\vec{n})$ that has a simple spectrum.

Let $P$ belong to $\mathcal{P}(4,3,3,3, \ldots, 3)$. By judiciously adding vertices to the boundary edges of $P$, we may regard $P$ as an element of $\mathcal{P}\left(n_{0}, n_{1}, n_{2}, n_{3}, \ldots, n_{k}\right)$, where $n_{0} \geq 4$ and $n_{i} \geq 3$ for $i>0$. Thus, it will suffice to prove that there exists some $P \in \mathcal{P}(4,3,3,3, \ldots, 3)$ such that $P$ has a simple spectrum. 
By Theorem 7.3 there exists a quadrilateral $Q$ with $k$ slits $\Sigma_{1}, \Sigma_{2}, \ldots, \Sigma_{k}$ so that $Q_{\Sigma_{1} \cup \Sigma_{2} \cup \cdots \cup \Sigma_{k}}$ has a simple spectrum. For each $i$, choose a point $p_{i}$ so that the convex hulls, $P_{i}$, of $\left\{p_{i}\right\} \cup \Sigma_{i}$ satisfy conditions (1) and (2) above. Let $m_{i}$ be the midpoint of $\Sigma_{i}$ and define the path $x_{i}(t)=t p_{i}+(1-t) m_{i}$. Define $P_{i}(t)$ to be the convex hull of $\left\{x_{i}\right\} \cup \Sigma_{i}$. Let $P(t)$ be the multiply connected polygon defined by $Q, P_{1}(t), P_{2}(t), \ldots, P_{k}(t)$. See Figure 3 Note that $P(t)$ is an element of $\mathcal{P}(4,3,3, \ldots, 3)$ for $t>0$ and that $P(0)$ corresponds to $\left(Q, \Sigma_{1}, \Sigma_{2}, \ldots, \Sigma_{n}\right)$.

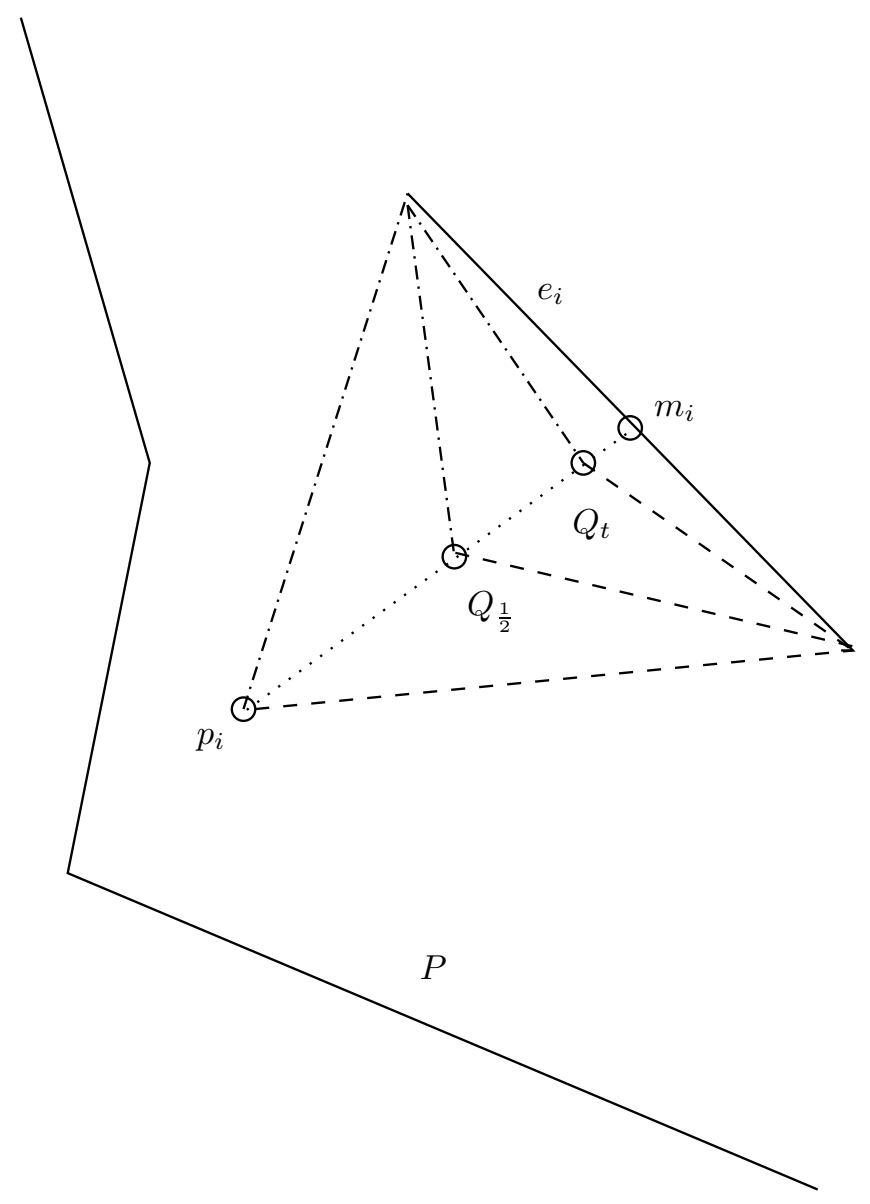

Figure 3. Perturbing a slit into a triangle

We define a linear path $t \mapsto f_{t}$ of piecewise linear homeomorphisms mapping $P(1 / 2)$ onto $P(t)$ for each $t \in[0,1 / 2]$. In particular, let $Q_{i}(t)$ be the quadrilateral $P_{i}(1) \backslash P_{i}(t)$, and let $f_{t}^{i}$ be the 'obvious' piecewise linear homeomorphism that maps $Q_{i}(1 / 2)$ onto $Q_{i}(t)$. Define $f_{t}: P(1 / 2) \rightarrow P(t)$ by

$$
f_{t}(x)=\left\{\begin{array}{cl}
x, & \text { if } x \notin \bigcup_{i} Q_{i}(1 / 2), \\
f_{t}^{i}(x), & \text { if } x \in Q_{i}(1 / 2) .
\end{array}\right.
$$


By Lemma 2.1 in HlrJdg09, the eigenvalues of $P(t)$ vary real-analytically in $t$. Since the spectrum of $P(0)$ is simple, there exists $t_{0}>0$ such that the spectrum of $P\left(t_{0}\right)$ is simple. The claim is proven.

\section{Appendix A. Mathieu functions}

For the convenience of the reader we provide basic facts about Mathieu functions that are used in the present paper. For additional information, we refer the reader to Mth68 and MrsFsh. Our approach is based on analytic perturbation theory. See Example VII.3.4 in [Kato].

Suppose that $\Delta \psi=E \cdot \psi$. A straightforward computation shows that

$$
-\left(\partial_{z}^{2}+\partial_{\theta}^{2}\right)\left(\psi \circ F_{t}\right)=t^{2} \cdot E \cdot\left(\cosh (z)^{2}-\cos ^{2}(\theta)\right) \cdot\left(\psi \circ F_{t}\right) .
$$

Let $h=t \sqrt{E}$. The method of separation of variables leads one to consider the operator

$$
A_{h}=-\frac{d^{2}}{d \theta^{2}}+h^{2} \cos ^{2}(\theta)
$$

acting selfadjointly on $L^{2}(\mathbb{R} /(2 \pi \mathbb{Z}), d \theta)$.

Proposition A.1 (Angular Mathieu functions). For each $i \in \mathbb{Z}$, there exist unique real-analytic paths $v_{k}: \mathbb{R} \rightarrow L^{2}(\mathbb{R} /(2 \pi \mathbb{Z}), d \theta)$ and $b_{k}: \mathbb{R} \rightarrow \mathbb{R}$ so that for each $h \in \mathbb{R}$ :

(a) $v_{k}(h)$ is an eigenfunction for $A_{h}$ with eigenvalue $b_{k}(h)$ :

$$
A_{h}\left(v_{k}(h)\right)=b_{k}(h) \cdot v_{k}(h) .
$$

(b) $v_{k}(h)$ has unit norm in $L^{2}(\mathbb{R} /(2 \pi \mathbb{Z}), d \theta)$.

(c) The span of $\left\{v_{k}(h) \mid k=0,1,2 \ldots\right\}$ is dense in $L^{2}(\mathbb{R} /(2 \pi \mathbb{Z}), d \theta)$.

(d) For $k$ odd,

$$
v_{k}(h)(\theta)=\pi^{-\frac{1}{2}} \cdot \sin (k \theta)+O\left(h^{2}\right),
$$

and for $k$ even,

$$
v_{k}(h)(\theta)=\pi^{-\frac{1}{2}} \cdot \cos (k \theta)+O\left(h^{2}\right) .
$$

(e) For $k$ odd,

$$
b_{k}(h)=k^{2}+2 h^{2}+O\left(h^{4}\right)
$$

and for $k$ even,

$$
b_{k}(h)=k^{2}+\frac{1}{2} h^{2}+O\left(h^{4}\right) .
$$

(f) For any $k$ and any $h$, we have $b_{k}(h) \geq b_{k}(0)$.

Remark A.2. The function $\theta \mapsto v_{k}(h)(\theta)$ is known classically as an angular Mathieu function. See for example [MrsFsh]. We will use both $v_{k}(h)$ and $v_{k, h}$ to denote this function of $\theta$.

Proof. The path $h \mapsto A_{h}$ is an analytic family of compactly resolved operators. Hence the existence of paths $v_{k}$ and $b_{k}$ satisfying (a), (b), and (c) follows from Theorem VII.3.9 on page 392 in Kato.

The $k^{2}$-eigenspace of $A_{0}=\partial_{\theta}^{2}$ is spanned by $\mathcal{B}_{k}=\{\sin (k \cdot \theta), \cos (k \cdot \theta)\}$. Let $P_{k}$ be the orthogonal projection onto this eigenspace. Let $\ddot{A}$ denote the second 
derivative of $h \mapsto A_{h}$ evaluated at $h=0$. One computes the matrix of $P_{k} \ddot{A} P_{k}$ with respect to the orthonormal basis $\mathcal{B}_{k}$ to be

$$
\left(\begin{array}{cc}
1 / 2 & 0 \\
0 & 2
\end{array}\right)
$$

The uniqueness of the paths as well as property (d) then follow from analytic perturbation theory [Kato. The derivative of $b_{k}$ is given by

$$
\frac{d}{d h} b_{i}(h)=2 h \int_{0}^{2 \pi} \cos ^{2}(\theta) v_{k, h}(\theta)^{2} d \theta .
$$

Thus $b_{k}$ is increasing for positive $h$ and decreasing for negative $h$ showing that $h=0$ is a global minimum.

Corollary A.3. Let $\psi$ be an eigenvector of $\Delta$ with Dirichlet (resp. Neumann) boundary condition on the slit. Then

$$
\psi(z, \theta)=\sum_{i} u_{k, h}(z) \cdot v_{k, h}(\theta),
$$

where

$$
v_{k, h}=v_{k}(h)
$$

are as above, and $u=u_{k, h}$ satisfies the ordinary differential equation

$$
-u^{\prime \prime}(z)+\left(b_{k}(h)-h^{2} \cosh ^{2}(z)\right) \cdot u(z)=0,
$$

with Dirichlet (resp. Neumann) boundary condition at $z=0$.

Proof. Each eigenfunction $\psi$ is smooth, and hence by Proposition A.1.

$$
\psi(z, \theta)=\sum_{i} u_{k, h}(z) \cdot v_{k, h}(\theta),
$$

where $h^{2}=t^{2} E$, and

$$
u_{k, h}(r)=\int_{0}^{2 \pi} \psi(z, \theta) \cdot v_{k, h}(\theta) d \theta .
$$

One obtains (20) by using (44) and integrating by parts.

\section{ACKNOWLEDGEMENT}

We thank the referee for valuable comments.

\section{REFERENCES}

[CdV82] Y. Colin de Verdière. Pseudo-laplaciens I, Ann. Institut Fourier, 32(3):275-286, 1982. MR688031 (84k:58221)

[CdV87] Y. Colin de Verdière. Construction de laplaciens dont une partie finie du spectre est donnée. Ann. Sci. École Norm. Sup. (4), 20(4):599-615, 1987. MR932800 (90d:58156)

[EMZ03] A. Eskin, H. Masur, and A. Zorich, Moduli spaces of abelian differentials: The principal boundary, counting problems, and the Siegel-Veech constants. Publ. Math. Inst. Hautes Études Sci. No. 97 (2003), 61-179. MR2010740 (2005b:32029)

[HlrJdg09] L. Hillairet and C. Judge. Generic spectral simplicity of polygons. Proc. Amer. Math. Soc. 137 (2009), 2139-2145. MR2480296(2009k:58065)

[MrsRbn38] P. M. Morse and P. J. Rubinstein, Diffraction of waves by ribbons and slits, Physical Review, 54, pp. 895-898, 1938.

[MrsFsh] P. M. Morse and H. Feshbach. Methods of theoretical physics. 2 volumes. McGraw-Hill Book Co., Inc., New York, 1953. MR0059774 (15:583h) 
[Mth68] É. Mathieu. Mémoire sur le mouvement vibratoire d'une membrane de forme elliptique. Journal de Mathématiques Pures et Appliquées, pp. 137-203, 1868.

[Jdg02] C. M. Judge. Tracking eigenvalues to the frontier of moduli space. II. Limits for eigenvalue branches. Geom. Funct. Anal., 12(1):93-120, 2002. MR1904559 (2004b:58051)

[Kato] T. Kato. Perturbation theory for linear operators. Springer-Verlag, Berlin, second edition, 1976. Grundlehren der Mathematischen Wissenschaften, Band 132. MR0407617 (53:11389)

[Gri] P. Grisvard. Elliptic problems in nonsmooth domains. Pitman, 1985. Monographs and Studies in Mathematics, 24. MR775683 (86m:35044)

[Wlp92] S. A. Wolpert. Spectral limits for hyperbolic surfaces. I, II. Invent. Math., 108(1), 1992, pp. 67-89, 91-129. MR.1156387 (93b:58160)

Laboratoire de Mathématiques Jean Leray, UMR CNRS 6629, Université de Nantes, 2 Rue de la Houssinière, BP 92 208, F-44 322 Nantes Cedex 3, France

E-mail address: Luc.Hillairet@math.univ-nantes.fr

Department of Mathematics, Indiana University, Bloomington, Indiana 47401

E-mail address: cjudge@indiana.edu 